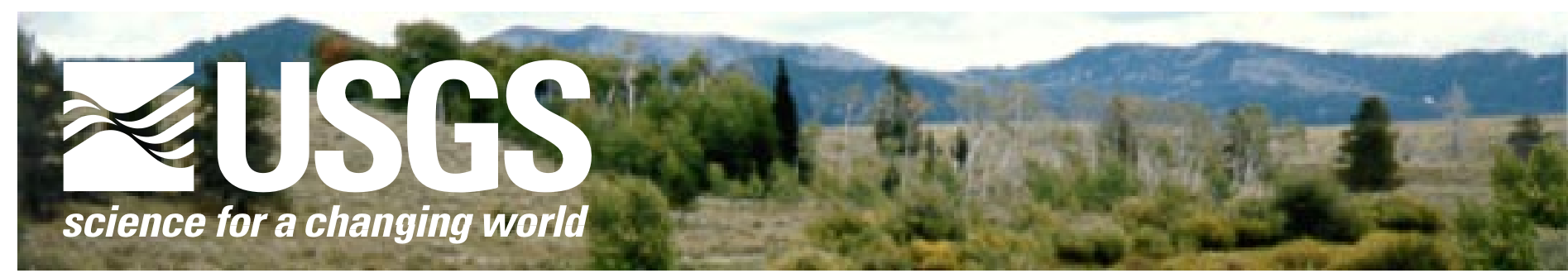

\title{
Nitrate Concentrations in Ground Water in the Henrys Fork Basin, Eastern Idaho
}

In 1998 and 1999, the U.S. Geological Survey (USGS) completed comprehensive studies of nitrate concentrations in ground water in the Henrys Fork Basin in eastern Idaho (fig. 1A). These studies were done in cooperation with the following agencies or groups: Idaho Division of Environmental Quality (DEQ), District 7 Health Department, Idaho Department of Water Resources (IDWR), Idaho Department of Agriculture (IDAG), Bureau of Reclamation, Henrys Fork Foundation, Fremont County, and Madison County. This Fact Sheet presents selected results of these investigations.

\section{Description of the Area}

The Henrys Fork Basin in parts of Teton, Madison, and Fremont Counties in Idaho comprises lower Henrys Fork (Menan Buttes to about Ashton, including the Fall River drainage), Teton River, and upper Henrys Fork drainages. Detailed descriptions of climate, geographic setting, land and water uses, geology, hydrology, and generalized water quality of the area are provided in reports listed in the "Selected References" section, back of this report.

Geology and hydrology of the basin are diverse and complex. Unconsolidated sediment varies in thickness from a few feet to hundreds of feet; thicknesses are greatest in areas from Menan Buttes to St. Anthony.
Sediment overlies variable thicknesses of volcanic rocks, and groundwater yielding zones exist in sediment and volcanic rock units. Regionally, ground water moves generally southward to southwestward but, in localized areas, ground-water movement can be northwestward or southeastward (Crosthwaite and others, 1970; Whitehead, 1978; Spinazola, 1994). Total well depths vary from tens of feet to hundreds of feet below land surface. Some well casings may be perforated or screened at one or more intervals or wells may be constructed with uncased boreholes to increase well yields.

Most ground water in the Henrys Fork Basin is nonthermal (temperature less than 29 degrees Celsius, ${ }^{\circ} \mathrm{C}$; 84 degrees Fahrenheit, ${ }^{\circ} \mathrm{F}$ ), but geothermal water (temperature greater than $29^{\circ} \mathrm{C}$ ) is present in many parts of the basin. On the basis of U.S. Environmental Protection Agency (EPA, 1996) standards for public water supplies, ground water in the Henrys Fork Basin is suitable for most public and private drinking-water uses. Ground water in this area is highly or very highly vulnerable to contamination (Rupert and others, 1991), and water in a few areas contains contaminants-components that can limit the water's suitability for use or can represent degradation of water quality.

Potential pathways of contaminant movement to ground-water zones include downward flushing of contaminants by infiltration of precipitation, floodwater, or applied irrigation water; flushing from soil and unsatur- ated rocks by seasonal variations in ground-water levels; leakage around or into well casings or boreholes (especially important in areas with thin layers of soil and sediment overlying volcanic rock); dumping into wells; backflushing to wells through water-supply systems; and transport from upgradient ground-water zones. Contamination from land and water uses can be localized (point source) or widespread (nonpoint source).

One of the most widespread contaminants in the Henrys Fork Basin is nitrate $\left(\mathrm{NO}_{3}\right)$. In this report, nitrate is reported as nitrogen $\left(\mathrm{NO}_{3}-\mathrm{N}\right.$ or nitrate-N) in milligrams per liter (mg/L, equivalent to parts per million).

\section{Nitrate-N Concentrations in Ground Water}

Concentrations of nitrate- $\mathrm{N}$ in Idaho prior to land and water development probably were less than $1 \mathrm{mg} / \mathrm{L}$. From 1995 through 1999, the median concentration (50th percentile) of nitrate- $\mathrm{N}$ in Idaho ground water was about $1.4 \mathrm{mg} / \mathrm{L}$, and the range of concentrations was $<0.05$ to $100 \mathrm{mg} / \mathrm{L}$. During the same period, the median nitrate-N concentration in the Henrys Fork Basin was $3.2 \mathrm{mg} / \mathrm{L}$, and the range of concentrations was $<0.05$ to $38 \mathrm{mg} / \mathrm{L}$.

Large nitrate- $\mathrm{N}$ concentrations primarily are of concern as a drinking-water health risk, and the maximum EPA (1996) limit for nitrate-N concentration in public water supplies is $10 \mathrm{mg} / \mathrm{L}$. Recommendations 
Figure 1A. Locations of ground-water sampling sites where nitrate (as nitrogen) concentrations are less than 5 milligrams per liter, and generalized directions of ground-water movement, Henrys Fork Basin, Idaho.

\section{EXPLANATION}

Upper Henrys Fork drainage

Teton River drainage

Lower Henrys Fork drainage

Generalized direction of groundwater movement (dashed where inferred)—Data from Crosthwaite and others (1970); Whitehead (1978); Spinazola (1994)

4 Sampling site where nitrate (as nitrogen) concentration was less than 5 milligrams per liter

Base from U.S. Geological Survey digital data, 1:500,000, 1980

Albers Equal-Area projection

Standard parallels $43^{\circ} 30^{\circ}, 47^{\circ} 30^{\prime}$, and $-114^{\circ} 00^{\prime}, 41^{\circ} 45^{\prime}$

No false easting or false northing
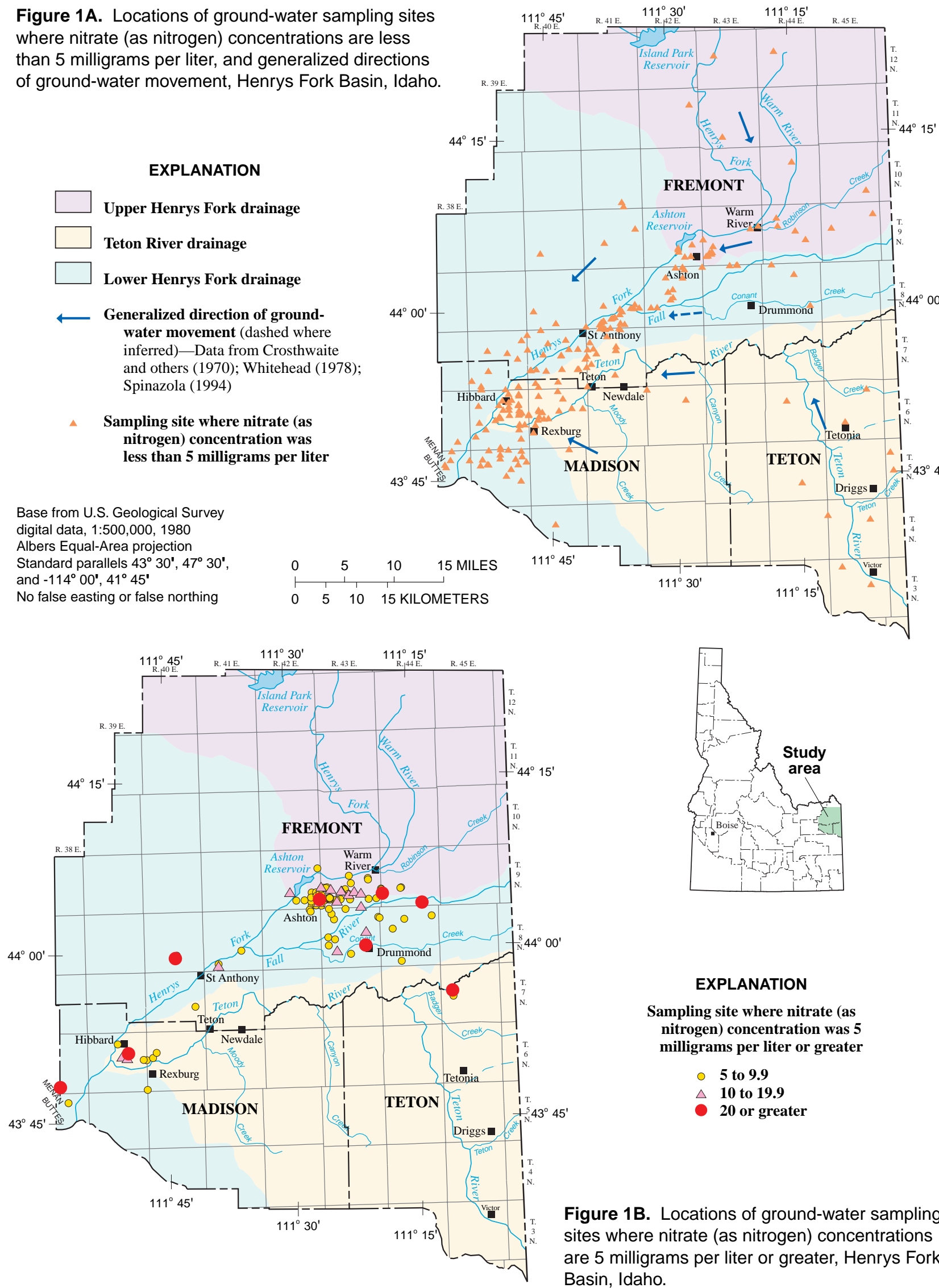

$11^{\circ} 45^{\prime}$
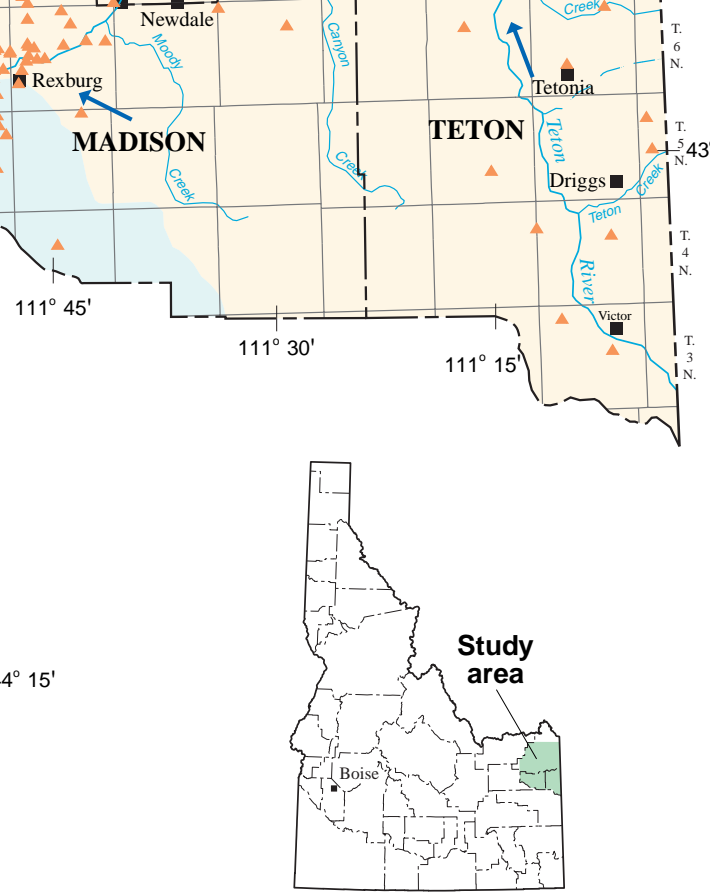

\section{EXPLANATION}

Sampling site where nitrate (as nitrogen) concentration was 5 milligrams per liter or greater

○ 5 to 9.9

$\triangle 10$ to 19.9

- 20 or greater

Figure 1B. Locations of ground-water sampling sites where nitrate (as nitrogen) concentrations are 5 milligrams per liter or greater, Henrys Fork Basin, Idaho. 
Table 1. Drinking-water recommendations for ranges of nitrate

[Modified from Mahler and others, 1990, p. 1; mg/L, milligrams per liter]

\begin{tabular}{|c|c|c|}
\hline $\begin{array}{l}\text { Nitrate as nitrogen } \\
\left(\mathrm{NO}_{3}-\mathrm{N} \text {, in } \mathrm{mg} / \mathrm{L}\right)^{1}\end{array}$ & $\begin{array}{l}\text { Nitrate as nitrate } \\
\left(\mathrm{NO}_{3}-\mathrm{NO}_{3} \text {, in } \mathrm{mg} / \mathrm{L}\right)^{2}\end{array}$ & Comments \\
\hline 11 to 20 & 45 to 88 & $\begin{array}{l}\text { Generally safe for human adult and livestock consumption. Do not use for human } \\
\text { infants. }\end{array}$ \\
\hline 21 to 40 & 89 to 176 & $\begin{array}{l}\text { Short-term use acceptable for human adult and all livestock consumption unless } \\
\text { food or feed sources are very high in nitrate. Long-term use could be risky. } \\
\text { Do not use for human infants. }\end{array}$ \\
\hline 41 to 100 & 177 to 440 & $\begin{array}{l}\text { Moderate to high risk for human adult and young livestock consumption. Proba- } \\
\text { bly acceptable for mature livestock if feed is low in nitrate. Do not use for } \\
\text { human infants. }\end{array}$ \\
\hline Over 100 & Over 440 & Do not use. \\
\hline
\end{tabular}

${ }^{1}$ Laboratory nitrate analysis reported as nitrogen $(\mathrm{N})$, in $\mathrm{mg} / \mathrm{L}$.

${ }^{2}$ Laboratory nitrate analysis reported as nitrate $\left(\mathrm{NO}_{3}\right)$, in $\mathrm{mg} / \mathrm{L}$.

for various ranges of nitrate- $\mathrm{N}$ in drinking water are shown in table 1.

Chronic, long-term health risks from consumption of water containing large nitrate concentrations are not fully understood. Short-term effects include the risk of methemoglobinemia (blue-baby syndrome, characterized by the reduced ability of blood to carry oxygen) to human infants, a small percentage of adults, and young livestock (Mahler and others, 1990, p. 1). Large concentrations of nitrate- $\mathrm{N}$ may be implicated with an increased incidence of nonHodgkin's lymphoma (Weisenburger, 1991, p. 309). Anomalous nitrate-N concentrations also imply active exchange of water from land surface to ground-water zones and the potential for water contamination by microorganisms or other chemical compounds (Parliman, 1998, p. 4).

\section{Ground-Water Analyses and Site Descriptions}

From 1995 through 1999, water samples were collected by the USGS or DEQ from 319 wells or springs in the Henrys Fork Basin and analyzed for nitrate- $\mathrm{N}$ concentrations ${ }^{1}$. For sites with multiple analyses, only the most recent analysis per site was used in this report. Complete site descrip- tions and water-quality data are available from the USGS District office in Boise, Idaho. Well locations, total well depth, and selected water-quality data are available in MS-EXCEL spreadsheet format at the Idaho District Web site http://idaho.usgs.gov/ until approximately February 2001.

Locations of ground-water sites are displayed in figures $1 \mathrm{~A}$ and $1 \mathrm{~B}$ according to ranges of nitrate- $\mathrm{N}$ concentrations. Concentrations in most water samples are relatively small (less than $5 \mathrm{mg} / \mathrm{L}$ ). In some parts of the study area, water contains moderately large nitrate- $\mathrm{N}$ concentrations (5 to $9.9 \mathrm{mg} / \mathrm{L}$ ) or concentrations exceeding the EPA (1996) maximum contaminant level of $10 \mathrm{mg} / \mathrm{L}$, a potential health risk for some drinkingwater purposes.

\section{Historical Nitrate-N Concentrations}

Nitrate-N data prior to about 1990 are sparsely distributed or clustered in the Henrys Fork Basin. Most historical nitrate- $\mathrm{N}$ concentrations were less than $5 \mathrm{mg} / \mathrm{L}$, but concentrations exceeding $20 \mathrm{mg} / \mathrm{L}$ were analyzed in water samples from wells east of Ashton and near Menan Buttes in the 1970 's. Nitrates could have been increasing since about the 1970's in

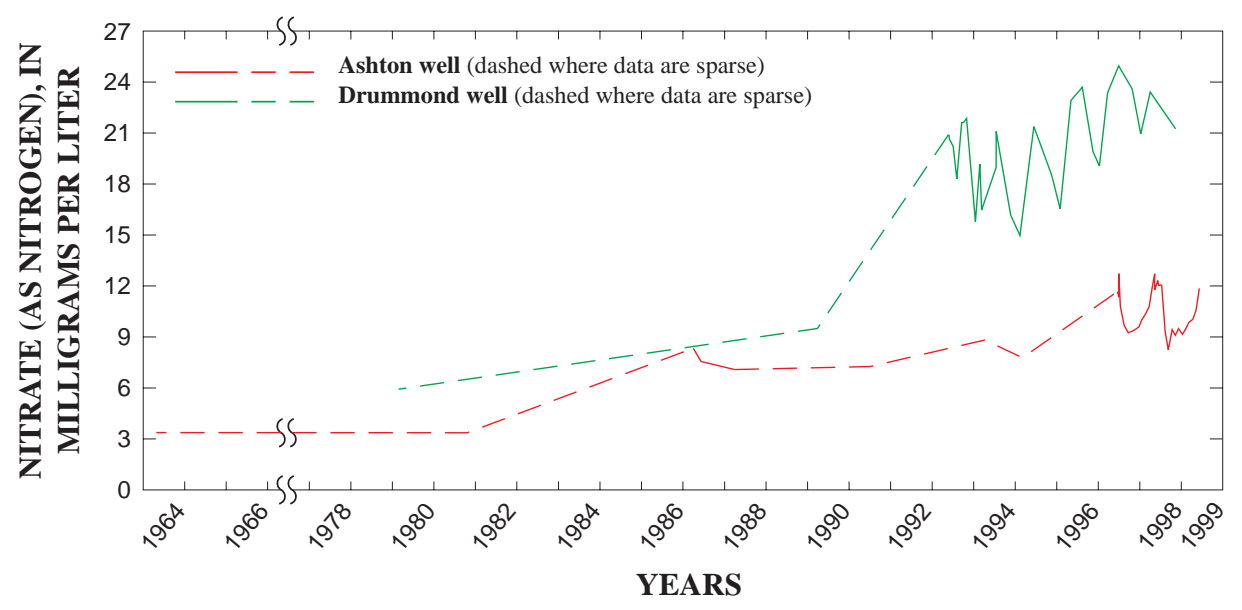

Figure 2. Changes in nitrate (as nitrogen) concentrations, 1964-99, in water samples from wells at Ashton and Drummond, Henrys Fork Basin, Idaho.

1USGS laboratory analyses are reported in units of nitrite plus nitrate as nitrogen $\left(\mathrm{NO}_{2}+\mathrm{NO}_{3}\right.$ as $\left.\mathrm{N}\right)$. DEQ laboratory analyses are reported in units of nitrate as nitrogen $\left(\mathrm{NO}_{3}\right.$ as $\left.\mathrm{N}\right)$. Concentrations of nitrite in ground water generally are negligible. For purposes of this report, USGS and DEQ analyses are comparable. 


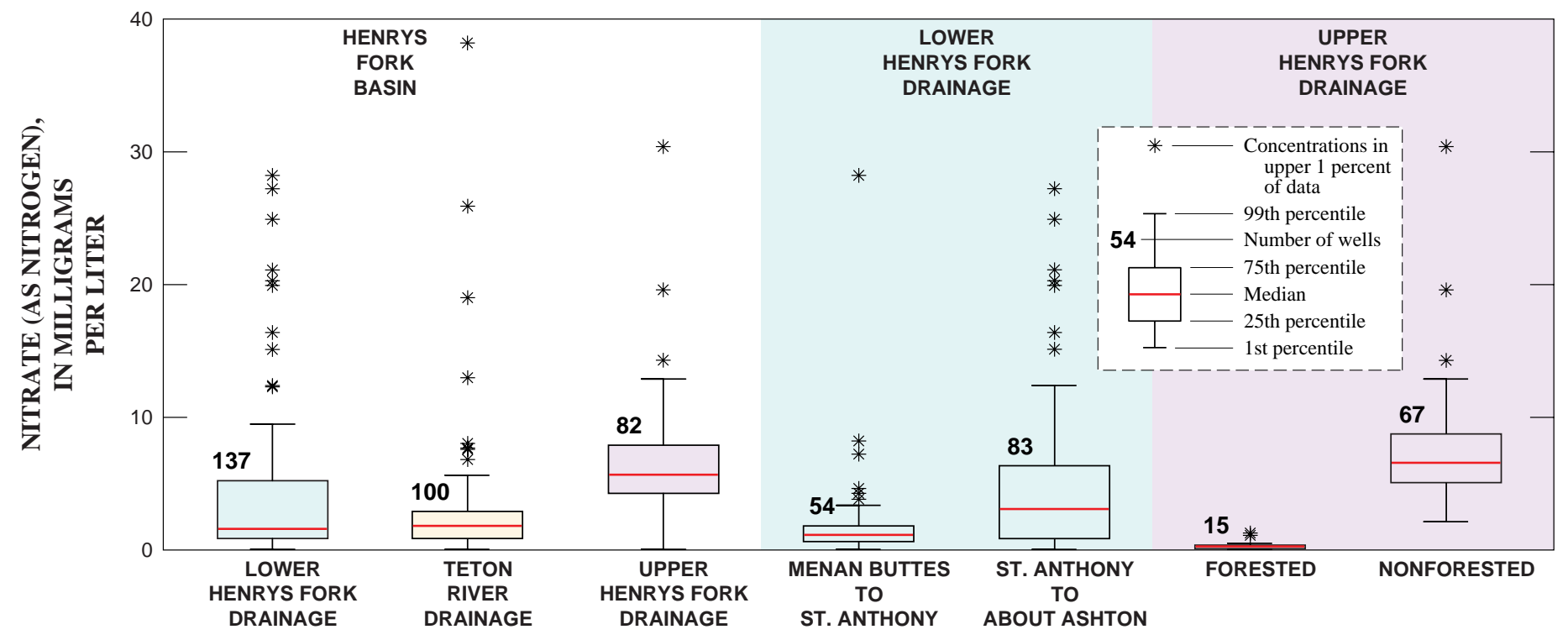

Figure 3. Nitrate (as nitrogen) data for wells in Henrys Fork Basin, Idaho.

areas where overall nitrate- $\mathrm{N}$ concentrations are currently large.

Changes in nitrate- $\mathrm{N}$ concentrations over time for water samples from wells at Drummond and Ashton are shown in figure 2. Total depth of both wells is about 300 feet, and hydrogeologic environments and nearby land-use histories are similar. Few nitrate- $\mathrm{N}$ data are available for either well from 1950 to about 1980, but the patterns of increasing nitrate-N concentrations are similar-relatively low nitrate-N concentrations until about 1980, then increasing concentrations to 1999 accompanied by seasonal fluctuations in concentrations each year. Causes for increasing concentrations currently are not fully understood.

\section{Statistical Summary of Nitrate-N Data}

Statistical summaries (boxplots) of nitrate-N concentrations for lower Henrys Fork, Teton River, and upper Henrys Fork drainages are compared in figure 3. Median (50th percentile) concentrations for lower Henrys Fork and Teton River drainages are similar $(1.6 \mathrm{mg} / \mathrm{L}$ and $1.9 \mathrm{mg} / \mathrm{L}$, respectively), but the median concentration for the upper Henrys Fork area is $5.7 \mathrm{mg} / \mathrm{L}$. Substantial differences are also evident in the distribution of nitrate- $\mathrm{N}$ concentrations within each drainage, as shown in the comparison of summaries for segments of the lower Henrys Fork drainageMenan Buttes to St. Anthony and St. Anthony to about Ashton. From Menan Buttes to St. Anthony, the median concentration is $1.1 \mathrm{mg} / \mathrm{L}$, and from St. Anthony to about Ashton, the median is $3.1 \mathrm{mg} / \mathrm{L}$. In the upper Henrys Fork drainage, large differences in concentrations are evident between areas with little or no landand water-use development (forested) and areas with extensive and varied land- and water-use development (nonforested). The median concentration for forested areas is $0.3 \mathrm{mg} / \mathrm{L}$, and the median concentration for nonforested areas is $6.6 \mathrm{mg} / \mathrm{L}$.

\section{Sources of Nitrogen}

Rupert (1996) estimated the amount of total nitrogen input by cattle manure, fertilizer, legume crops (such as alfalfa and beans), precipitation, and domestic septic systems for counties in the upper Snake River Basin, eastern Idaho and western Wyoming. The estimates were used to rank the input of nitrogen by source and determine the amount of total nitrogen potentially available to ground and surface water through leaching and runoff. Results showed that about 45 percent of the input was from fertilizers, 29 percent from cattle manure, 19 percent from legume crops, 6 percent from precipitation, and less than 1 percent from domestic septic systems. Input from cattle manure, fertilizers, and legume crops varied widely among counties, reflecting differences in land-use practices such as cropping patterns and numbers of dairies and feedlots. In the Henrys Fork Basin, Rupert indicated that fertilizers were the major source of nitrogen input. Manure and crops were secondary sources, and precipitation and domestic septic systems were minor sources of nitrogen input.

\section{Nitrogen Isotope Data}

One method for estimating sources of nitrogen to ground water is analysis for stable isotopes of nitrogen. Stable isotopes are nonradioactive forms of an element. Nitrogen isotopes ${ }^{15} \mathrm{~N}$ and ${ }^{14} \mathrm{~N}$ constitute an isotope pair, and lab analyses determine the ratio of the abundance of the heavier isotope $\left({ }^{15} \mathrm{~N}\right)$ to that of the lighter isotope $\left({ }^{14} \mathrm{~N}\right)$ in water. The major potential sources of nitrogen contamination commonly have characteristic ${ }^{15} \mathrm{~N} / 14 \mathrm{~N}$ ratios (expressed in 


\begin{tabular}{|lc|}
\hline $\begin{array}{l}\text { Table 2. Nitrogen isotope ratios of } \\
\text { common sources of nitrate in water }\end{array}$ \\
\hline $\begin{array}{lc}\text { Potential contaminant } \\
\text { ratio source }\end{array}$ & $\begin{array}{c}\mathbf{1 5}^{\mathbf{N}} /{ }^{14} \mathbf{N} \\
\text { (permil) }\end{array}$ \\
\hline $\begin{array}{l}\text { Precipitation } \\
\text { Commercial fertilizer }\end{array}$ & -3 \\
Organic nitrogen in soil & -4 to +4 \\
Animal waste & +4 to +9 \\
Greater than +10
\end{tabular}

permil units, or parts per thousand). Typical ${ }^{15} \mathrm{~N} /{ }^{14} \mathrm{~N}$ ratio values for nitrogen sources are presented in table 2 (Seiler, 1996, p. 12). Total well depth, nitrate- $\mathrm{N}$, and nitrogen isotope ratio data for recent ground-water samples in the Henrys Fork Basin are shown in table 3.

Additional nitrogen isotope analyses are available through DEQ for areas near Ashton where intermediate nitrogen isotope ratio values in water could indicate a mixture of nitrogen sources (D. Yashan, DEQ, written commun., 1999).

\section{Other Sources of Nitrate-N Data}

The comprehensive study of nitrate- $\mathrm{N}$ in ground water in the Henrys Fork Basin has been completed, but there are ongoing ground-water quality programs through several agencies and organizations, including USGS, DEQ, District 7 Health Department, IDAG, and consultants. For private wellowners, nitrate-N analyses are available through local analytical laboratories. Information on water quality, health risks, laboratory analyses for private wellowners, and water-treatment options is avail- able through DEQ and District 7 Health Department agencies.

\section{Additional Information Needed}

Effects of several hydrogeologic and environmental factors on groundwater quality are not well understood at this time. Additional information is needed in the following areas: (1) causes of increasing nitrate- $\mathrm{N}$ concentrations since about 1980; (2) effects of well construction on contamination of multiple wateryielding zones; (3) seasonal change in nitrate-N concentrations; (4) seasonal change in water levels and directions of ground-water movement in major water-yielding zones, particularly in the Fall River drainage; (5) comparative age dates of water in major water-yielding zones to help determine rates of contaminant movement; and (6) groundwater/surface-water relations in areas where nitrate- $\mathrm{N}$ concentrations are large. Large nitrate- $\mathrm{N}$ concentrations in ground water could contribute to increased nitrate- $\mathrm{N}$ concentrations in surface water, potentially resulting in deterioration of surface-water quality.

\section{Acknowledgments}

Special thanks are extended to wellowners in the study area for access to property and permission to collect water samples from their wells. Additional thanks are extended to personnel from the City of Ashton, Lockheed Martin Idaho Technol- ogies Company, Brown and Caldwell, URS Greiner Woodward Clyde, and Ricks College for information and unpublished data for the area.

—by D.J. Parliman

\section{Selected References}

Brown and Caldwell, 1994, J.R. Simplot Company Soilbuilders Facility, Drummond, Idaho: Boise, Brown and Caldwell, unpublished report on file in the Boise office of the U.S. Geological Survey, 9 p., 7 attachments.

Bureau of Reclamation, 1977, Groundwater studies-Henrys Fork, Teton River area, Fremont and Madison Counties, Idaho: Boise, Bureau of Reclamation, $27 \mathrm{p}$.

Clark, G.M., Maret, T.R., Rupert, M.G., Maupin, M.A., Low, W.H., and Ott, D.S., 1998, Water quality in the upper Snake River Basin, Idaho and Wyoming, 19921995: U.S. Geological Survey Circular 1160, $35 \mathrm{p}$.

Crosthwaite, E.G., (1960), Geology and hydrology of the Rexburg area, Idaho: U.S. Geological Survey unpublished paper on file in the Boise office of the U.S. Geological Survey, $17 \mathrm{p}$.

1979, Chemical analyses of ground water related to geothermal investigations in the Teton River area, eastern Idaho: U.S. Geological Survey Open-File Report 79-687, 14 p.

Crosthwaite, E.G., Mundorff, M.J., and Walker, E.H., 1970, Ground-water aspects of the lower Henrys Fork region, eastern Idaho: U.S. Geological Survey Water-Supply Paper 1879-C, 22 p., 1 pl.

Embree, G.F., Lovell, M.D., and Doherty, D.J., 1978, Drilling data from Sugar City exploration well, Madison County, Idaho: U.S. Geological Survey Open-File Report 78-1095, 1 pl.

Gellenbeck, D.J., 1994, Isotopic compositions and sources of nitrate in ground water from western Salt River Valley, Arizona:

Table 3. Total well depth, dissolved nitrate (as nitrogen), and nitrogen isotope ratio data ${ }^{1}$ for selected wells, Henrys Fork Basin, Idaho

[mg/L, milligrams per liter]

\begin{tabular}{|c|c|c|c|c|}
\hline Water sample source & Sample date & $\begin{array}{l}\text { Depth of well, } \\
\text { total (feet) }\end{array}$ & $\begin{array}{c}\text { Nitrate, } \\
\text { dissolved } \\
(\mathrm{mg} / \mathrm{L} \text { as } \mathrm{N})\end{array}$ & $\begin{array}{c}15 \mathrm{~N} / 14 \mathrm{~N} \\
\text { stable isotope ratio } \\
\text { (permil) }\end{array}$ \\
\hline 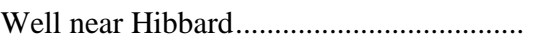 & 06-15-99 & 240 & 25.9 & 2.80 \\
\hline 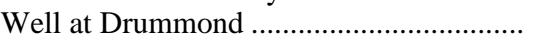 & $06-30-99$ & 310 & 21.1 & 8.30 \\
\hline \multirow[t]{2}{*}{ 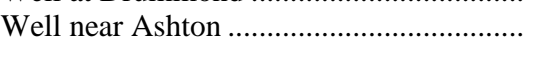 } & $10-06-98$ & 60 & 18.5 & 9.80 \\
\hline & $06-25-99$ & 60 & 30.4 & 10.10 \\
\hline
\end{tabular}

${ }^{1}$ Analyses from the U.S. Geological Survey National Water-Quality Laboratory, Denver, Colorado. Nitrogen isotopes, ${ }^{15} \mathrm{~N} /{ }^{14} \mathrm{~N}$ as nitrate and ammonia. 
U.S. Geological Survey Water-Resources Investigations Report 94-4063, 53 p.

Ham, H.H., 1968, Replacement ground water supply - first phase, Lower Teton Division, Teton Basin Project, Idaho: Boise, Bureau of Reclamation, $28 \mathrm{p}$.

Haskett, G.I., 1972, Groundwater investigations of the Rexburg Bench-second phase, Lower Teton Division, Teton Basin Project, Idaho: Boise, Bureau of Reclamation, $21 \mathrm{p}$.

Howarth, R.B., 1999, Assessment of sources of elevated nitrate in ground water in northwest Ada County, Idaho, using environmental isotopes: Boise, Idaho Division of Environmental Quality, Boise Regional Office, Ground Water Quality Technical Report no. 11, 50 p., 2 apps.

Idaho Department of Water Resources, 1998, Henrys Fork and Mud Lake areas (hydrologic data): Boise, unpublished map report on file in Boise office of the U.S. Geological Survey, $1 \mathrm{pl}$.

Jorgensen Engineering and Land Surveying, P.C., 1999, City of Ashton-water supply system nitrate mitigation study (draft): Jackson, Wyo., unpublished, 42 p.

Kilburn, Chabot, 1964, Ground water in the upper part of the Teton Valley, Teton Counties, Idaho and Wyoming: U.S. Geological Survey Water-Supply Paper 1789, 60 p., 4 pl.

Kirkham, V.R.D., 1925, Press bulletin concerning geologic conditions, oil possibilities, and drilling progress in Teton County, Idaho: Idaho Bureau of Mines and Geology Press Bulletin no. 15, 4 p.

Mahler, R.L., Porter, Ernestine, and Taylor, Roy, 1990, Quality water for Idahonitrate and groundwater: Moscow, University of Idaho, College of Agriculture, Current Information Series no. 872, 2 p.

Maupin, M.A., 1995, Water-quality assessment of the upper Snake River Basin, Idaho and western Wyoming - environmental setting, 1980-92: U.S. Geological Survey Water-Resources Investigations Report 94-4221, 35 p.
Meachum, Teresa, 1999, Nitrates in groundwater-a study of levels in southeast Idaho: Idaho Falls, University of Idaho, unpublished M.S. thesis, 48 p., 2 apps.

Parliman, D.J., 1983, Reconnaissance of ground-water quality, eastern Snake River Basin, Idaho: U.S. Geological Survey Water-Resources Investigations Report 82-4004, 100 p., 4 pls.

1998, Ground-water quality in northern Ada County, lower Boise River Basin, Idaho, 1985-96: U.S. Geological Survey Fact Sheet FS-054-98, 6 p.

Ray, H.A., Kjelstrom, L.C., Crosthwaite, E.G., and Low, W.H., 1978, The flood in southeastern Idaho from the Teton Dam failure of June 5, 1976, with a section on ground water fluctuations and on quality of surface and ground water: U.S. Geological Survey Open-File Report 77-765, 48 p., 3 pls.

Rupert, M.G., 1994, Analysis of data on nutrients and organic compounds in ground water in the upper Snake River Basin, Idaho and western Wyoming, 1980-91: U.S. Geological Survey Water-Resources Investigations Report 94-4135, 40 p.

- 1996, Major sources of nitrogen input and loss in the upper Snake River Basin, Idaho and western Wyoming, 1990: U.S. Geological Survey Water-Resources Investigations Report 96-4008, 15 p. 1997, Nitrate $\left(\mathrm{NO}_{2}+\mathrm{NO}_{3}-\mathrm{N}\right)$ in ground water of the upper Snake River Basin, Idaho and western Wyoming, 1991-95: U.S. Geological Survey WaterResources Investigations Report 97-4174, $47 \mathrm{p}$.

1998, Probability of detecting atrazine/desethyl-atrazine and elevated concentrations of nitrate $\left(\mathrm{NO}_{2}+\mathrm{NO}_{3}-\mathrm{N}\right)$ in ground water in the Idaho part of the upper Snake River Basin: U.S. Geological Survey Water-Resources Investigations Report 98-4203, 32 p., 1 pl.

Rupert, Michael, Dace, Tana, Maupin, Molly, and Wicherski, Bruce, 1991, Ground water vulnerability assessment-Snake River
Plain, southern Idaho: Boise, Idaho Department of Health and Welfare, Division of Environmental Quality, 25 p.

Seiler, R.L., 1996, Methods for identifying sources of nitrogen contamination of ground water in valleys in Washoe County, Nevada: U.S. Geological Survey OpenFile Report 96-461, 29 p.

Spinazola, J.M., 1994, Geohydrology and simulation of flow and water levels in the aquifer system in the Mud Lake area of the eastern Snake River Plain, eastern Idaho: U.S. Geological Survey Water-Resources Investigations Report 93-4227, 78 p.

Spinazola, J.M., Tungate, A.M., and Rogers, T.L., 1992, Geohydrologic and chemical data from wells in the Mud Lake area, eastern Idaho, 1988-91: U.S. Geological Survey Open-File Report 92-133, 92 p.

Tungate, A.M., 1995, Hydrographs of water levels in observation wells in Idaho, 194493: U.S. Geological Survey Open-File Report 95-458, 422 p.

U.S. Environmental Protection Agency, 1996, Drinking water regulations and health advisories: U.S. Environmental Protection Agency, Office of Water, 822-B-96-002, $18 \mathrm{p}$.

URS Greiner Woodward Clyde, 1998, Western Farm Service facility, Parker, Idaho: Seattle, URS Greiner Woodward Clyde, unpublished report on file in Boise office of the U.S. Geological Survey.

Weisenburger, D.D., 1991, Potential health consequences of ground-water contamination by nitrates in Nebraska, in Bogardi, I., Kuzelka, R.D., and Ennenga, W.G., Nitrate contamination - exposure, consequence, and control: Berlin, Springer-Verlag, p. 309-315.

Whitehead, R.L., 1978, Water resources of the upper Henrys Fork Basin in eastern Idaho: Boise, Idaho Department of Water Resources Water Information Bulletin no. 46,48 p., 6 pls.

For more information about U.S. Geological Survey programs, publications, and resources in Idaho, visit our Web site at http://idaho.usgs.gov

or contact:

District Chief

Photo at left: Warm River Springs on Henrys Fork northeast of Ashton, Idaho (Photo by D.J. Parliman, 1998).
230 Collins Road

Boise, ID 83702-4520
U.S. Geological Survey, WRD 
( < = less than; -- = data not available; NO2+NO3 = nitrite plus nitrate; MG/L AS N = milligrams per liter as nitrogen; latitude and longitude in degrees, minutes, and seconds based on North American datum of 1927 (NAD27);

$\mathrm{N}-15 / \mathrm{N}-14$ = nitrogen-15/nitrogen-14 as nitrate and ammonia; USGS = U. S. Geological Survey;

$\mathrm{DEQ}=$ Idaho Division of Environmental Quality)

DEQ

USGS ANALYSES

ANALYSES

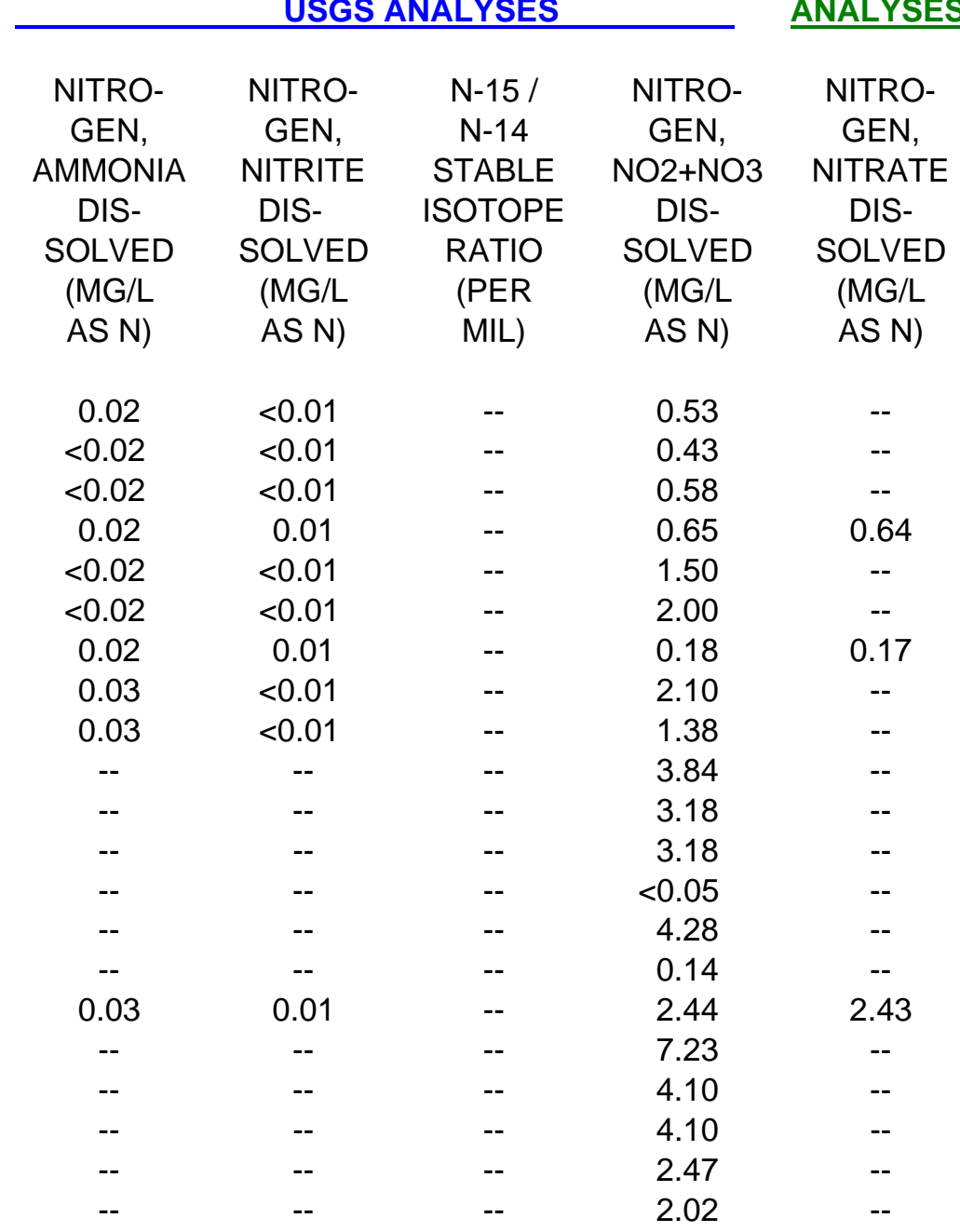




\begin{tabular}{|c|c|c|c|c|c|c|c|c|c|}
\hline \multirow[t]{2}{*}{ 05N 39E 03CDB1 } & 434725 & 1115126 & 06/15/99 & 68 & -- & -- & -- & 1.98 & -- \\
\hline & & & $06 / 15 / 99$ & 68 & -- & -- & -- & 1.97 & -- \\
\hline 05N 39E 04BCA1 & 434749 & 1115249 & 06/29/99 & 60 & -- & -- & -- & 2.68 & -- \\
\hline 05N 39E 05DDD1 & 434743 & 1114305 & 06/16/99 & 88 & -- & -- & -- & 1.03 & -- \\
\hline 05N 39E 07BAC1 & 434659 & 1115510 & 06/29/99 & 50 & -- & -- & -- & 0.66 & -- \\
\hline 05N 39E 08ABC1 & 434701 & 1115336 & 06/16/99 & 35 & -- & -- & -- & 0.86 & -- \\
\hline 05N 39E 08CDB1 & 434630 & 1115352 & 06/16/99 & 25 & -- & -- & -- & 0.68 & -- \\
\hline 05N 39E 09CBC1 & 434635 & 1115302 & 06/16/99 & 175 & -- & -- & -- & 0.24 & -- \\
\hline 05N 39E 09CBD1 & 434634 & 1115253 & 06/16/99 & 60 & -- & -- & -- & 0.76 & -- \\
\hline 05N 39E 10BAC1 & 434704 & 1115127 & 06/15/99 & 132 & -- & -- & -- & 0.97 & -- \\
\hline 05N 39E 10BDB1 & 434657 & 1115127 & 08/04/98 & -- & -- & -- & -- & -- & 1.87 \\
\hline \multirow[t]{2}{*}{ 05N 39E 11AAC1 } & 434700 & 1114935 & 08/31/95 & 72 & $<0.02$ & $<0.01$ & -- & 1.60 & -- \\
\hline & & & $06 / 21 / 99$ & 72 & $<0.02$ & $<0.01$ & -- & 1.61 & -- \\
\hline \multirow[t]{2}{*}{ 05N 39E 12CBA1 } & 434631 & 1114918 & 08/31/95 & 60 & 0.02 & $<0.01$ & -- & 2.50 & -- \\
\hline & & & $06 / 14 / 99$ & 60 & $<0.02$ & $<0.01$ & -- & 2.11 & -- \\
\hline 05N 39E 12DAA1 & 434644 & 1114817 & 06/15/99 & 81 & -- & -- & -- & 3.07 & -- \\
\hline 05N 39E 14BAD1 & 434607 & 1115010 & 06/15/99 & 100 & -- & -- & -- & 2.94 & -- \\
\hline 05N 39E 14BAD2 & & & 06/15/99 & -- & -- & -- & -- & 2.31 & -- \\
\hline \multirow[t]{3}{*}{ 05N 39E 15DDD1 } & 434532 & 1115045 & 10/17/95 & 61 & -- & -- & -- & -- & 1.50 \\
\hline & & & 10/21/98 & 61 & -- & -- & -- & -- & 1.40 \\
\hline & & & 06/17/99 & 61 & -- & -- & -- & 1.55 & -- \\
\hline \multirow[t]{2}{*}{ 05N 39E 24BDD1 } & 434505 & 1114900 & 08/11/97 & 60 & $<0.02$ & $<0.01$ & -- & 2.78 & -- \\
\hline & & & 06/22/99 & 60 & -- & -- & -- & 1.47 & -- \\
\hline 05N 44E 27ABC1 & 434410 & 1111500 & 08/09/96 & 180 & 0.02 & $<0.01$ & -- & 0.38 & -- \\
\hline \multirow[t]{2}{*}{ 05N 46E 07BDA1 } & 434635 & 1110412 & 08/06/97 & 160 & 0.03 & $<0.01$ & -- & 0.80 & -- \\
\hline & & & 08/06/97 & 160 & 0.02 & $<0.01$ & -- & 0.81 & -- \\
\hline \multirow[t]{3}{*}{ 05N 46E 19AAB1 } & 434501 & 1110353 & 07/11/95 & 206 & 0.02 & $<0.01$ & -- & 0.73 & -- \\
\hline & & & 09/14/98 & 206 & 0.02 & 0.01 & -- & 0.70 & 0.69 \\
\hline & & & 07/27/99 & 206 & $<0.02$ & $<0.01$ & -- & 0.50 & -- \\
\hline 06N 38E 34BCC1 & 434822 & 1115835 & 05/14/98 & -- & -- & -- & -- & -- & 28.20 \\
\hline \multirow[t]{2}{*}{ 06N 38E 35ABA1 } & 434838 & 1115633 & 08/30/95 & 303 & $<0.02$ & $<0.01$ & -- & 0.18 & -- \\
\hline & & & 06/29/99 & 303 & $<0.02$ & $<0.01$ & -- & 0.16 & -- \\
\hline 06N 39E 01CBB1 & 435233 & 1114858 & 06/17/99 & -- & -- & -- & -- & 0.91 & -- \\
\hline 06N 39E 01CCC2 & 435241 & 1114900 & 06/17/99 & 86 & -- & -- & -- & 1.29 & -- \\
\hline 06N 39E 02CDC1 & 435211 & 1114954 & 06/17/99 & 80 & -- & -- & -- & 4.71 & -- \\
\hline 06N 39E 03ADD1 & 435243 & 1115015 & 06/15/98 & 100 & -- & -- & -- & -- & 1.90 \\
\hline 06N 39E 07ADD1 & 435144 & 1115355 & $08 / 11 / 97$ & 120 & 0.02 & $<0.01$ & -- & 0.91 & -- \\
\hline
\end{tabular}




\begin{tabular}{|c|c|c|c|c|c|c|c|c|c|}
\hline & & & 06/29/99 & 120 & -- & -- & -- & 0.86 & -- \\
\hline 06N 39E 09ADA1 & 435145 & 1115127 & $06 / 16 / 99$ & 82 & -- & -- & -- & 3.54 & -- \\
\hline \multirow[t]{2}{*}{ 06N 39E 10AAD1 } & 435159 & 1115016 & 08/04/97 & 79 & 0.02 & $<0.01$ & -- & 3.24 & -- \\
\hline & & & $06 / 22 / 99$ & 79 & -- & -- & -- & 2.23 & -- \\
\hline 06N 39E 10BABA1 & 435209 & 1115100 & 06/15/98 & 90 & -- & -- & -- & -- & 3.10 \\
\hline 06N 39E 10BBB5 & 435208 & 1115121 & $06 / 15 / 98$ & 80 & -- & -- & -- & -- & 5.00 \\
\hline 06N 39E 10CDD1 & 435120 & 1115056 & 06/16/99 & 100 & -- & -- & -- & 1.73 & -- \\
\hline \multirow[t]{2}{*}{ 06N 39E 10DAAD2 } & 435136 & 1115015 & 06/15/99 & 267 & -- & -- & -- & 2.26 & -- \\
\hline & & & $06 / 15 / 99$ & 267 & -- & -- & -- & 2.16 & -- \\
\hline \multirow[t]{7}{*}{ 06N 39E 10DDDD1 } & 435120 & 1115015 & 01/23/95 & 79 & -- & -- & -- & -- & 4.56 \\
\hline & & & 02/22/96 & 79 & -- & -- & -- & -- & 7.08 \\
\hline & & & $02 / 18 / 97$ & 79 & -- & -- & -- & -- & 6.52 \\
\hline & & & $01 / 06 / 98$ & 79 & -- & -- & -- & -- & 6.85 \\
\hline & & & 05/07/98 & 79 & -- & -- & -- & -- & 8.24 \\
\hline & & & 07/28/98 & 79 & -- & -- & -- & -- & 10.90 \\
\hline & & & 08/24/98 & 79 & -- & -- & -- & -- & 6.81 \\
\hline \multirow[t]{3}{*}{ 06N 39E 10DDDD2 } & & & $01 / 04 / 98$ & 260 & -- & 0.09 & -- & -- & 2.23 \\
\hline & & & $11 / 18 / 98$ & 260 & -- & -- & -- & -- & 2.67 \\
\hline & & & 06/15/99 & 260 & -- & -- & -- & 2.70 & -- \\
\hline 06N 39E 11ADD1 & 435147 & 1114904 & 06/17/99 & 80 & -- & -- & -- & 3.21 & -- \\
\hline 06N 39E 11DAA1 & 435142 & 1114905 & 06/16/99 & 85 & -- & -- & -- & -- & 1.20 \\
\hline 06N 39E 13BBB1 & 435115 & 1114900 & 06/16/98 & 80 & -- & -- & -- & -- & 2.00 \\
\hline 06N 39E 13BCA1 & 435103 & 1114900 & 06/16/99 & 218 & -- & -- & -- & 1.78 & -- \\
\hline 06N 39E 13BCD1 & 435058 & 1114855 & 06/03/98 & 90 & -- & -- & -- & -- & 2.40 \\
\hline 06N 39E 13BDB1 & 435102 & 1114842 & $06 / 15 / 98$ & 127 & -- & -- & -- & -- & 4.00 \\
\hline \multirow[t]{2}{*}{ 06N 39E 13DAAA1 } & 435053 & 1114752 & 09/23/98 & 70 & -- & -- & -- & -- & 0.11 \\
\hline & & & 06/17/99 & 70 & -- & -- & -- & 0.44 & -- \\
\hline 06N 39E 13DAAD1 & 435048 & 1114752 & 06/17/99 & 70 & -- & -- & -- & 0.55 & -- \\
\hline 06N 39E 13DADC1 & 435043 & 1114815 & $06 / 17 / 99$ & 75 & -- & -- & -- & 8.05 & -- \\
\hline \multirow[t]{3}{*}{ 06N 39E 13DADD1 } & 435041 & 1114752 & 04/23/98 & -- & -- & -- & -- & -- & 6.42 \\
\hline & & & $11 / 19 / 98$ & -- & -- & -- & -- & -- & 10.70 \\
\hline & & & $06 / 17 / 99$ & -- & -- & -- & -- & 8.03 & -- \\
\hline \multirow[t]{5}{*}{ 06N 39E 14BBCC1 } & 435107 & 1115013 & 03/23/98 & 240 & -- & -- & -- & -- & 28.60 \\
\hline & & & 03/30/98 & 240 & -- & -- & -- & -- & 28.40 \\
\hline & & & 04/16/98 & 240 & -- & -- & -- & -- & 25.50 \\
\hline & & & 06/23/98 & 240 & -- & -- & -- & -- & 21.20 \\
\hline & & & $10 / 08 / 98$ & 240 & $<0.02$ & $<0.01$ & -- & 27.10 & -- \\
\hline
\end{tabular}




\begin{tabular}{|c|c|c|c|c|c|c|c|c|c|}
\hline & & & 06/15/99 & 240 & -- & -- & 2.80 & 25.90 & -- \\
\hline 06N 39E 14BCB1 & 435103 & 1115013 & 06/16/99 & 90 & -- & -- & -- & 13.00 & -- \\
\hline 06N 39E 14CBB1 & 435052 & 1115012 & 06/16/98 & 80 & -- & -- & -- & -- & 1.60 \\
\hline 06N 39E 14DAA1 & 435052 & 1114904 & 06/15/98 & 260 & -- & -- & -- & -- & 2.80 \\
\hline 06N 39E 15BAD1 & 435112 & 1115050 & 06/17/99 & -- & -- & -- & -- & 19.00 & -- \\
\hline 06N 39E 15CBC1 & 435045 & 1115125 & 06/22/99 & 100 & -- & -- & -- & 3.41 & -- \\
\hline 06N 39E 15CCB1 & 435037 & 1115124 & $12 / 30 / 98$ & 100 & -- & -- & -- & -- & 3.23 \\
\hline 06N 39E 15CCB2 & 435038 & 1115117 & $04 / 21 / 98$ & 102 & -- & -- & -- & -- & 2.70 \\
\hline 06N 39E 16DCC1 & 435027 & 1115155 & 08/08/97 & 100 & 0.02 & $<0.01$ & -- & 2.33 & -- \\
\hline \multirow[t]{2}{*}{ 06N 39E 19AAD1 } & 435015 & 1115352 & 06/15/99 & 108 & -- & -- & -- & 1.14 & -- \\
\hline & & & 06/15/99 & 108 & -- & -- & -- & 1.14 & -- \\
\hline \multirow[t]{5}{*}{ 06N 39E 20DCC1 } & 434939 & 1115306 & 08/30/95 & 89 & $<0.02$ & $<0.01$ & -- & 3.10 & -- \\
\hline & & & 08/12/96 & 89 & 0.02 & 0.01 & -- & 2.60 & 2.59 \\
\hline & & & 08/08/97 & 89 & 0.02 & $<0.01$ & -- & 2.59 & -- \\
\hline & & & 08/19/98 & 89 & 0.03 & $<0.01$ & -- & 2.95 & -- \\
\hline & & & 06/21/99 & 89 & $<0.02$ & $<0.01$ & -- & 2.54 & -- \\
\hline 06N 39E 23DDDD1 & 434935 & 1114904 & 01/27/98 & -- & -- & -- & -- & -- & 0.48 \\
\hline \multirow[t]{3}{*}{ 06N 39E 24ACC1 } & 435000 & 1114822 & 09/06/95 & 97 & $<0.02$ & $<0.01$ & -- & $<0.05$ & -- \\
\hline & & & 09/06/95 & 97 & $<0.02$ & $<0.01$ & -- & $<0.05$ & -- \\
\hline & & & 06/14/99 & 97 & $<0.02$ & $<0.01$ & -- & $<0.05$ & -- \\
\hline 06N 39E 24BCCC1 & 435002 & 1114900 & 02/23/98 & -- & -- & -- & -- & -- & 1.84 \\
\hline 06N 39E 24CBB1 & 434958 & 1114900 & 06/16/99 & 64 & -- & -- & -- & 0.26 & -- \\
\hline 06N 39E 25CCD1 & 434846 & 1114851 & 06/16/99 & 80 & -- & -- & -- & 2.29 & -- \\
\hline 06N 39E 30ABB1 & 434903 & 1115420 & 04/27/98 & 44 & -- & -- & -- & -- & 0.42 \\
\hline \multirow[t]{2}{*}{ 06N 39E 34CCBB2 } & 434803 & 1115124 & 06/16/99 & 160 & -- & -- & -- & 1.59 & -- \\
\hline & & & 06/16/99 & 160 & -- & -- & -- & 1.56 & -- \\
\hline 06N 39E 34CCC1 & 434753 & 1115122 & 06/16/99 & 94 & -- & -- & -- & 2.74 & -- \\
\hline 06N 39E 36DAD1 & 434805 & 1114751 & 06/16/99 & 62 & -- & -- & -- & 5.63 & -- \\
\hline 06N 40E 03CDD1 & 435210 & 1114340 & 06/17/99 & 62 & -- & -- & -- & 2.28 & -- \\
\hline 06N 40E 04AAD1 & 435251 & 1114416 & 06/16/99 & 200 & -- & -- & -- & 3.12 & -- \\
\hline 06N 40E 05CBC1 & 435228 & 1114636 & 06/16/99 & 67 & -- & -- & -- & 0.86 & -- \\
\hline 06N 40E 08AAA1 & 435118 & 1114639 & 06/29/99 & 83 & -- & -- & -- & 5.53 & -- \\
\hline \multirow[t]{2}{*}{ 06N 40E 11CDCC1 } & 435119 & 1114237 & 09/11/98 & 52 & 0.02 & $<0.01$ & -- & 2.77 & -- \\
\hline & & & 06/22/99 & 52 & -- & -- & -- & 0.87 & -- \\
\hline 06N 40E 13BAA1 & 435116 & 1114117 & 06/17/99 & 141 & -- & -- & -- & 0.74 & -- \\
\hline 06N 40E 16AAC1 & 435108 & 1114411 & 06/03/98 & 69 & -- & -- & -- & -- & 1.50 \\
\hline 06N 40E 17BBC1 & 435107 & 1114635 & 08/03/98 & 195 & -- & $<0.01$ & -- & -- & 1.50 \\
\hline
\end{tabular}




\begin{tabular}{|c|c|c|c|c|c|c|c|c|c|}
\hline \multirow[t]{2}{*}{ 06N 40E 17BDB1 } & 435102 & 1114612 & 09/23/98 & 104 & -- & -- & -- & -- & 0.96 \\
\hline & & & $06 / 15 / 99$ & 104 & -- & -- & -- & 1.15 & -- \\
\hline 06N 40E 17CBC1 & 435039 & 1114636 & 03/18/98 & 42 & -- & -- & -- & -- & 1.60 \\
\hline 06N 40E 17DCC1 & 435028 & 1114601 & 06/22/99 & 64 & -- & -- & -- & 2.18 & -- \\
\hline \multirow[t]{3}{*}{ 06N 40E 18AAA1 } & 435114 & 1114639 & 08/06/97 & 59 & 0.02 & $<0.01$ & -- & 0.13 & -- \\
\hline & & & 06/22/99 & 59 & -- & -- & -- & 0.45 & -- \\
\hline & & & 06/22/99 & 59 & -- & -- & -- & 0.45 & -- \\
\hline 06N 40E 18DBB1 & 435052 & 1114706 & 06/01/98 & -- & -- & -- & -- & -- & 7.59 \\
\hline 06N 40E 18DDD1 & 435029 & 1114529 & 04/09/98 & 69 & -- & -- & -- & -- & 1.32 \\
\hline 06N 40E 19AAB2 & 435023 & 1114646 & $12 / 08 / 98$ & 250 & -- & -- & -- & -- & 3.96 \\
\hline \multirow[t]{2}{*}{ 06N 40E 19DBA1 } & 434955 & 1114705 & 06/16/99 & 175 & -- & -- & -- & 0.90 & -- \\
\hline & & & 06/16/99 & 175 & -- & -- & -- & 0.91 & -- \\
\hline 06N 40E 30BDD1 & 434916 & 1114720 & 06/24/99 & 61 & -- & -- & -- & 0.96 & -- \\
\hline 06N 42E 06BCB1 & 435244 & 1113326 & 08/06/97 & 500 & 0.02 & $<0.01$ & -- & 1.19 & -- \\
\hline \multirow[t]{2}{*}{ 06N 42E 10ADA1 } & 435145 & 1112845 & 08/31/95 & 975 & $<0.02$ & $<0.01$ & -- & 0.84 & -- \\
\hline & & & 06/22/99 & 975 & $<0.02$ & $<0.01$ & -- & 0.96 & -- \\
\hline \multirow[t]{2}{*}{ 06N 44E 09CCA2 } & 435123 & 1111632 & 07/11/95 & 700 & $<0.02$ & $<0.01$ & -- & 1.00 & -- \\
\hline & & & 07/30/99 & 700 & $<0.02$ & $<0.01$ & -- & 2.32 & -- \\
\hline 06N 45E 02CDB1 & 435212 & 1110649 & $08 / 14 / 96$ & -- & 0.03 & 0.01 & -- & 0.08 & 0.07 \\
\hline 06N 45E 28BBC1 & 434922 & 1110932 & 08/09/96 & 110 & 0.02 & $<0.01$ & -- & 0.85 & -- \\
\hline 07N 39E 01CDD1 & 435723 & 1114829 & 06/22/99 & 110 & -- & -- & -- & 1.08 & -- \\
\hline 07N 39E 08DDD1 & 435632 & 1115240 & 06/17/99 & 124 & -- & -- & -- & 0.41 & -- \\
\hline 07N 39E 12CCC1 & 435633 & 1114747 & 06/22/99 & 72 & -- & -- & -- & 1.03 & -- \\
\hline 07N 39E 14DDA1 & 435547 & 1114902 & 06/22/99 & 144 & -- & -- & -- & 3.02 & -- \\
\hline 07N 39E 15ADA1 & 435618 & 1115016 & 06/29/99 & 160 & -- & -- & -- & 0.88 & -- \\
\hline 07N 39E 29AAA1 & 435446 & 1115237 & 06/16/99 & 200 & -- & -- & -- & 0.32 & -- \\
\hline 07N 39E 30CDC1 & 435356 & 1115439 & 06/17/99 & 160 & -- & -- & -- & 0.67 & -- \\
\hline 07N 39E 32CBA1 & 435327 & 1115332 & 06/17/99 & 159 & -- & -- & -- & 3.38 & -- \\
\hline \multirow[t]{2}{*}{ 07N 39E 32CCA1 } & 435312 & 1115332 & 08/30/95 & 55 & 0.06 & $<0.01$ & -- & 2.80 & -- \\
\hline & & & 06/21/99 & 55 & $<0.02$ & $<0.01$ & -- & 4.65 & -- \\
\hline \multirow[t]{2}{*}{ 07N 39E 32CCA2 } & 435310 & 1115330 & 08/11/97 & 120 & $<0.02$ & $<0.01$ & -- & 1.78 & -- \\
\hline & & & 06/24/99 & 120 & -- & -- & -- & 1.52 & -- \\
\hline 07N 39E 34DCD2 & 435307 & 1115035 & 06/17/99 & 76 & -- & -- & -- & 0.65 & -- \\
\hline 07N 39E 36CBC1 & 435322 & 1114900 & 06/17/99 & 79 & -- & -- & -- & 1.06 & -- \\
\hline \multirow[t]{3}{*}{ 07N 40E 05BAA1 } & 435814 & 1114607 & 09/23/98 & 157 & -- & -- & -- & -- & 1.50 \\
\hline & & & 09/23/98 & 157 & -- & -- & -- & -- & 1.10 \\
\hline & & & 06/23/99 & 157 & -- & -- & -- & 1.13 & -- \\
\hline
\end{tabular}




\begin{tabular}{|c|c|c|c|c|c|c|c|c|c|}
\hline 07N 40E 06BCC1 & 435750 & 1114747 & 06/22/99 & 85 & -- & -- & -- & 1.05 & -- \\
\hline \multirow{3}{*}{ 07N 40E 09BCB1 } & 435704 & 1114518 & 09/22/98 & 70 & 0.12 & -- & -- & -- & 0.58 \\
\hline & & & 09/22/98 & 70 & -- & -- & -- & -- & 0.09 \\
\hline & & & 06/23/99 & 70 & -- & -- & -- & 0.32 & -- \\
\hline 07N 40E 10AAA1 & 435719 & 1114307 & 06/23/99 & 100 & -- & -- & -- & 0.67 & -- \\
\hline 07N 40E 11CCD1 & 435630 & 1114246 & 06/22/99 & 80 & -- & -- & -- & 0.89 & -- \\
\hline 07N 40E 12CCD1 & 435632 & 1114140 & 06/22/99 & 160 & -- & -- & -- & 1.42 & -- \\
\hline 07N 40E 13DDC1 & 435537 & 1114048 & 06/22/99 & 180 & -- & -- & -- & 1.46 & -- \\
\hline \multirow[t]{2}{*}{ 07N 40E 15CAA1 } & 435558 & 1114345 & 09/23/98 & 130 & -- & -- & -- & -- & 0.33 \\
\hline & & & 06/15/99 & 130 & -- & -- & -- & 0.39 & -- \\
\hline 07N 40E 15CAA2 & 435559 & 1114342 & 06/15/99 & 150 & -- & -- & -- & 0.97 & -- \\
\hline \multirow[t]{3}{*}{ 07N 40E 17ACC1 } & 435605 & 1114554 & 09/22/98 & 80 & -- & -- & -- & -- & 0.59 \\
\hline & & & 06/22/99 & 80 & -- & -- & -- & 0.46 & -- \\
\hline & & & 06/22/99 & 80 & -- & -- & -- & 0.46 & -- \\
\hline 07N 40E 19DDD1 & 435448 & 1114648 & 06/22/99 & 60 & -- & -- & -- & 0.48 & -- \\
\hline 07N 40E 22CDD1 & 435446 & 1114340 & 06/23/99 & 140 & -- & -- & -- & 0.68 & -- \\
\hline 07N 40E 24BCC1 & 435515 & 1114147 & 06/23/99 & 80 & -- & -- & -- & 8.24 & -- \\
\hline 07N 40E 24DDA1 & 435453 & 1114040 & 06/17/99 & 130 & -- & -- & -- & 1.15 & -- \\
\hline 07N 40E 25BBA1 & 435444 & 1114139 & 06/23/99 & 100 & -- & -- & -- & 1.53 & -- \\
\hline 07N 40E 25BBB1 & 435444 & 1114146 & 06/29/99 & 90 & -- & -- & -- & 1.29 & -- \\
\hline 07N 40E 29AAD1 & 435438 & 1114535 & 06/29/99 & 130 & -- & -- & -- & 2.87 & -- \\
\hline 07N 40E 30BAB1 & 435445 & 1114725 & $01 / 21 / 98$ & 50 & -- & -- & -- & -- & 0.96 \\
\hline \multirow[t]{2}{*}{ 07N 40E 30BAB2 } & 435445 & 1114731 & 06/16/99 & 50 & -- & -- & -- & 0.80 & -- \\
\hline & & & 06/16/99 & 50 & -- & -- & -- & 0.78 & -- \\
\hline 07N 40E 31ADB1 & 435337 & 1114655 & 06/17/99 & 100 & -- & -- & -- & 1.25 & -- \\
\hline 07N 40E 32CBCC1 & 435316 & 1114635 & 09/11/98 & 74 & 0.03 & $<0.01$ & -- & 1.62 & -- \\
\hline 07N 40E 34BBC1 & 435341 & 1114411 & 06/17/99 & 90 & -- & -- & -- & 0.85 & -- \\
\hline 07N 40E 36DDA1 & 435311 & 1114039 & 05/04/98 & 121 & -- & -- & -- & -- & 0.99 \\
\hline 07N 41E 04CCB1 & 435732 & 1113807 & 06/23/99 & 180 & -- & -- & -- & 0.83 & -- \\
\hline 07N 41E 05AAA1 & 435810 & 1113815 & 06/15/99 & 203 & -- & -- & -- & 1.28 & -- \\
\hline \multirow[t]{2}{*}{ 07N 41E 07BCC1 } & 435702 & 1114012 & 09/23/98 & 170 & -- & -- & -- & -- & 1.50 \\
\hline & & & 06/17/99 & 170 & -- & -- & -- & 1.78 & -- \\
\hline 07N 41E 10CCB1 & 435637 & 1113702 & 06/29/99 & 65 & -- & -- & -- & 0.13 & -- \\
\hline 07N 41E 15ABC1 & 435617 & 1113702 & 06/29/99 & 78 & -- & -- & -- & 1.84 & -- \\
\hline 07N 41E 16AAD1 & 435618 & 1113705 & 06/29/99 & 60 & -- & -- & -- & 2.24 & -- \\
\hline 07N 41E 18ABA1 & 435629 & 1113947 & 06/23/99 & 152 & -- & -- & -- & 0.54 & -- \\
\hline 07N 41E 18BBD1 & 435626 & 1114029 & 06/24/99 & 170 & -- & -- & -- & 1.76 & -- \\
\hline
\end{tabular}




\begin{tabular}{|c|c|c|c|c|c|c|c|c|c|}
\hline 07N 41E 18CBC1 & 435551 & 1114035 & 06/22/99 & 100 & -- & -- & -- & 0.52 & -- \\
\hline 07N 41E 18DAA1 & 435603 & 1113927 & 06/23/99 & 200 & -- & -- & -- & 1.85 & -- \\
\hline 07N 41E 19CAA1 & 435509 & 1114005 & 06/23/99 & 120 & -- & -- & -- & 1.19 & -- \\
\hline \multirow[t]{7}{*}{ 07N 45E 17CBA1 } & 435557 & 1111034 & 07/11/95 & 220 & 0.02 & $<0.01$ & -- & 17.00 & -- \\
\hline & & & 09/22/95 & 220 & $<0.02$ & $<0.01$ & -- & 0.07 & -- \\
\hline & & & 09/22/95 & 220 & $<0.02$ & $<0.01$ & -- & 12.00 & -- \\
\hline & & & 08/27/96 & 220 & $<0.02$ & $<0.01$ & -- & 11.00 & -- \\
\hline & & & 08/27/96 & 220 & $<0.02$ & $<0.01$ & -- & 11.00 & -- \\
\hline & & & 08/05/97 & 220 & 0.04 & $<0.01$ & -- & 35.40 & -- \\
\hline & & & 09/16/98 & 220 & 0.04 & 0.02 & -- & 38.20 & 38.20 \\
\hline 07N 45E 20BAB1 & 435530 & 1111026 & $08 / 14 / 96$ & 422 & 0.04 & 0.01 & -- & 7.70 & 7.69 \\
\hline 08N 40E 27BAC1 & 435947 & 1114353 & 07/19/96 & 165 & 0.03 & $<0.01$ & -- & 5.50 & -- \\
\hline \multirow[t]{3}{*}{ 08N 40E 27CBB1 } & 435919 & 1114412 & 07/19/96 & 113 & 0.04 & 0.01 & -- & 23.00 & 23.00 \\
\hline & & & 06/24/99 & 113 & -- & -- & -- & 27.70 & -- \\
\hline & & & 06/29/99 & 113 & $<0.02$ & $<0.01$ & 0.60 & 27.20 & -- \\
\hline \multirow[t]{2}{*}{ 08N 40E 30ABA1 } & 435954 & 1114656 & 08/10/95 & 73 & $<0.02$ & $<0.01$ & -- & 1.20 & -- \\
\hline & & & 06/24/99 & 73 & $<0.02$ & $<0.01$ & -- & 2.96 & -- \\
\hline \multirow[t]{2}{*}{ 08N 40E 33ADA1 } & 435848 & 1114415 & 09/22/98 & 167 & 0.07 & -- & -- & -- & 2.60 \\
\hline & & & 06/23/99 & 167 & -- & -- & -- & 0.88 & -- \\
\hline \multirow[t]{2}{*}{ 08N 41E 20DAC1 } & 440013 & 1113825 & 08/01/96 & 265 & $<0.02$ & $<0.01$ & -- & 1.30 & -- \\
\hline & & & 06/29/99 & 265 & -- & -- & -- & 2.00 & -- \\
\hline 08N 41E 22DDA1 & 440003 & 1113557 & 06/29/99 & 75 & -- & -- & -- & 3.28 & -- \\
\hline 08N 41E 22DDC1 & 440003 & 1113601 & 06/29/99 & 42 & -- & -- & -- & 8.78 & -- \\
\hline 08N 41E 23DCC1 & 435957 & 1113506 & 06/23/99 & -- & -- & -- & -- & 0.77 & -- \\
\hline 08N 41E 24CBC1 & 440015 & 1113436 & 06/24/99 & 65 & -- & -- & -- & 0.33 & -- \\
\hline 08N 41E 24CDD1 & 435957 & 1113405 & 06/15/99 & 59 & -- & -- & -- & 0.35 & -- \\
\hline 08N 41E 26AAA1 & 435955 & 1113442 & 06/23/99 & 57 & -- & -- & -- & 0.56 & -- \\
\hline 08N 41E 27ABB1 & 435954 & 1113625 & 06/17/99 & 55 & -- & -- & -- & 0.26 & -- \\
\hline 08N 41E 27ACA1 & 435940 & 1113610 & 06/29/99 & 92 & -- & -- & -- & 2.35 & -- \\
\hline 08N 41E 27CCB1 & 435912 & 1113704 & 06/23/99 & 258 & -- & -- & -- & 1.55 & -- \\
\hline 08N 41E 27DBB1 & 435926 & 1113627 & 06/30/99 & 240 & -- & -- & -- & 0.74 & -- \\
\hline 08N 41E 28DDC1 & 435408 & 1113715 & 06/23/99 & 240 & -- & -- & -- & 0.85 & -- \\
\hline 08N 41E 28DDC2 & 435908 & 1113720 & 06/23/99 & 59 & -- & -- & -- & 3.83 & -- \\
\hline 08N 41E 31CBC1 & 435826 & 1114036 & 06/22/99 & 190 & -- & -- & -- & 0.90 & -- \\
\hline \multirow[t]{3}{*}{ 08N 41E 32ABC1 } & 435856 & 1113850 & 09/01/95 & 148 & $<0.02$ & $<0.01$ & -- & 9.50 & -- \\
\hline & & & 08/01/96 & 148 & $<0.02$ & $<0.01$ & -- & 5.30 & -- \\
\hline & & & 08/07/97 & 148 & $<0.02$ & $<0.01$ & -- & 11.70 & -- \\
\hline
\end{tabular}




\begin{tabular}{|c|c|c|c|c|c|c|c|c|c|}
\hline & & & $06 / 22 / 99$ & 148 & -- & -- & -- & 6.90 & -- \\
\hline & & & 07/30/99 & 148 & $<0.02$ & $<0.01$ & -- & 5.24 & -- \\
\hline \multirow[t]{3}{*}{ 08N 41E 32ABC2 } & 435855 & 1113850 & 08/07/98 & 50 & 0.04 & 0.11 & -- & 22.90 & 22.80 \\
\hline & & & 08/07/98 & 50 & 0.03 & 0.11 & -- & 23.70 & 23.60 \\
\hline & & & $06 / 22 / 99$ & 50 & $<0.02$ & $<0.01$ & -- & 19.90 & -- \\
\hline 08N 41E 32ACC1 & 435846 & 1113843 & $06 / 22 / 99$ & 220 & -- & -- & -- & 1.30 & -- \\
\hline 08N 41E 32ADB1 & 435852 & 1113825 & $06 / 24 / 99$ & 75 & -- & -- & -- & 1.88 & -- \\
\hline 08N 41E 32ADB2 & 435852 & 1113830 & $06 / 24 / 99$ & 240 & -- & -- & -- & 1.40 & -- \\
\hline 08N 41E 32CAA1 & 435835 & 1113856 & $06 / 24 / 99$ & 250 & -- & -- & -- & 1.13 & -- \\
\hline 08N 41E 32CDD1 & 435814 & 1113855 & $06 / 24 / 99$ & 195 & -- & -- & -- & 0.84 & -- \\
\hline 08N 41E 32DCD1 & 435819 & 1113838 & $12 / 13 / 98$ & -- & -- & -- & -- & -- & 0.84 \\
\hline 08N 41E 32DDC1 & 435814 & 1113825 & $06 / 22 / 99$ & 240 & -- & -- & -- & 0.96 & -- \\
\hline \multirow[t]{2}{*}{ 08N 41E 33ABB2 } & 435904 & 1113731 & $07 / 14 / 95$ & -- & 0.02 & $<0.01$ & -- & 0.82 & -- \\
\hline & & & $06 / 23 / 99$ & -- & $<0.02$ & $<0.01$ & -- & 0.87 & -- \\
\hline 08N 41E 33CDD1 & 435815 & 1113740 & 06/24/99 & -- & -- & -- & -- & 1.14 & -- \\
\hline 08N 41E 34ACC1 & 435840 & 1113625 & 05/27/98 & -- & -- & -- & -- & -- & 1.07 \\
\hline 08N 41E 34CDC1 & 435813 & 1113636 & $06 / 24 / 99$ & 60 & -- & -- & -- & 0.92 & -- \\
\hline 08N 41E 34DAA1 & 435833 & 1113554 & $06 / 24 / 99$ & 102 & -- & -- & -- & 0.37 & -- \\
\hline 08N 41E 34DCC1 & 435807 & 1113626 & $06 / 24 / 99$ & 155 & -- & -- & -- & 0.79 & -- \\
\hline \multirow[t]{3}{*}{ 08N 42E 01AAA1 } & 440322 & 1112616 & 07/18/97 & 60 & -- & -- & -- & -- & 3.68 \\
\hline & & & 06/08/98 & 60 & 0.05 & 0.02 & -- & 5.61 & 5.60 \\
\hline & & & $10 / 07 / 98$ & 60 & $<0.02$ & $<0.01$ & -- & 4.12 & -- \\
\hline \multirow[t]{2}{*}{ 08N 42E 02AAD1 } & 440318 & 1112727 & 07/18/97 & 60 & -- & -- & -- & -- & 5.34 \\
\hline & & & 06/09/98 & 60 & 0.03 & 0.01 & -- & 5.76 & 5.74 \\
\hline 08N 42E 02BCA1 & 440311 & 1112826 & $06 / 15 / 99$ & 720 & -- & -- & -- & 1.63 & -- \\
\hline 08N 42E 03AAA1 & 440324 & 1112840 & 06/30/99 & 40 & -- & -- & -- & 4.88 & -- \\
\hline 08N 42E 03BAB1 & 440322 & 1112931 & $06 / 30 / 99$ & 82 & -- & -- & -- & 4.84 & -- \\
\hline \multirow[t]{2}{*}{ 08N 42E 09BAB1 } & 440232 & 1113045 & 08/07/97 & 173 & $<0.02$ & $<0.01$ & -- & 2.63 & -- \\
\hline & & & $06 / 24 / 99$ & 173 & -- & -- & -- & 3.85 & -- \\
\hline 08N 42E 10AAA1 & 440228 & 1112841 & $06 / 29 / 99$ & 420 & -- & -- & -- & 4.36 & -- \\
\hline 08N 42E 16AAD1 & 440128 & 1112955 & $06 / 29 / 99$ & 114 & -- & -- & -- & 3.68 & -- \\
\hline 08N 42E 16BDD1 & 440116 & 1113030 & 09/30/98 & 92 & -- & -- & -- & -- & 3.58 \\
\hline 08N 42E 19DCD1 & 435956 & 1113238 & $06 / 23 / 99$ & 100 & -- & -- & -- & 0.87 & -- \\
\hline 08N 42E 20CDC1 & 435956 & 1113154 & 06/23/99 & 125 & -- & -- & -- & 0.27 & -- \\
\hline 08N 42E $21 \mathrm{AAA} 1$ & 440046 & 1112955 & 06/29/99 & 182 & -- & -- & -- & 3.06 & -- \\
\hline 08N 42E $21 \mathrm{ABD} 1$ & 440035 & 1113014 & $06 / 24 / 99$ & 145 & -- & -- & -- & 1.45 & -- \\
\hline $08 \mathrm{~N} 42 \mathrm{E} 30 \mathrm{BCC} 1$ & 435931 & 1113126 & $06 / 23 / 99$ & 132 & -- & -- & -- & 1.68 & -- \\
\hline
\end{tabular}




\begin{tabular}{|c|c|c|c|c|c|c|c|c|c|}
\hline 08N 43E 01DDB1 & 440238 & 1111915 & 06/30/99 & 266 & -- & -- & -- & 5.16 & -- \\
\hline \multirow[t]{2}{*}{ 08N 43E 01DDD1 } & 440234 & 1111905 & 08/12/97 & 340 & 0.03 & $<0.01$ & -- & 5.41 & -- \\
\hline & & & 06/30/99 & 340 & -- & -- & -- & 5.55 & -- \\
\hline 08N 43E 05BCD1 & 440302 & 1112455 & 06/30/99 & 82 & -- & -- & -- & 6.08 & -- \\
\hline 08N 43E 05CDC1 & 440233 & 1112438 & 06/30/99 & 200 & -- & -- & -- & 8.98 & -- \\
\hline \multirow[t]{2}{*}{ 08N 43E 14ABB1 } & 440135 & 1112046 & 07/22/97 & -- & -- & -- & -- & -- & 11.40 \\
\hline & & & 06/30/99 & -- & -- & -- & -- & 12.30 & -- \\
\hline \multirow[t]{2}{*}{ 08N 43E 18DDB1 } & 440059 & 1112524 & 07/18/97 & -- & -- & -- & -- & -- & 6.90 \\
\hline & & & 06/30/99 & -- & -- & -- & -- & 8.23 & -- \\
\hline 08N 43E 19DAB1 & 440016 & 1112522 & 06/30/99 & 162 & -- & -- & -- & 8.94 & -- \\
\hline 08N 43E 20BAA1 & 440047 & 1112435 & 06/30/99 & 102 & -- & -- & -- & 9.48 & -- \\
\hline \multirow[t]{2}{*}{ 08N 43E 20DCC1 } & 435958 & 1112421 & 07/22/97 & 320 & -- & -- & -- & -- & 10.30 \\
\hline & & & 08/11/98 & 320 & 0.06 & $<0.01$ & -- & 15.10 & -- \\
\hline \multirow[t]{19}{*}{ 08N 43E 23DBC1 } & 440010 & 1112044 & 01/11/95 & 310 & -- & -- & -- & -- & 18.70 \\
\hline & & & $01 / 11 / 95$ & 310 & -- & -- & -- & -- & 20.90 \\
\hline & & & 05/23/95 & 310 & -- & -- & -- & -- & 15.80 \\
\hline & & & 08/15/95 & 310 & -- & -- & -- & -- & 14.50 \\
\hline & & & 12/20/95 & 310 & -- & -- & -- & -- & 21.20 \\
\hline & & & 02/29/96 & 310 & -- & -- & -- & -- & 19.90 \\
\hline & & & 05/30/96 & 310 & -- & -- & -- & -- & 18.20 \\
\hline & & & 08/15/96 & 310 & -- & -- & -- & -- & 16.20 \\
\hline & & & $11 / 21 / 96$ & 310 & -- & -- & -- & -- & 22.80 \\
\hline & & & 03/04/97 & 310 & -- & -- & -- & -- & 23.60 \\
\hline & & & 06/10/97 & 310 & -- & -- & -- & -- & 19.70 \\
\hline & & & 08/07/97 & 310 & -- & -- & -- & -- & 18.80 \\
\hline & & & 10/20/97 & 310 & -- & -- & -- & -- & 23.30 \\
\hline & & & 01/29/98 & 310 & -- & -- & -- & -- & 24.90 \\
\hline & & & 06/01/98 & 310 & -- & -- & -- & -- & 23.50 \\
\hline & & & 08/17/98 & 310 & -- & -- & -- & -- & 20.80 \\
\hline & & & $11 / 12 / 98$ & 310 & -- & -- & -- & -- & 23.30 \\
\hline & & & 06/30/99 & 310 & 0.06 & 0.02 & 8.30 & 21.10 & 21.10 \\
\hline & & & 06/30/99 & 310 & -- & -- & -- & 19.80 & -- \\
\hline 08N 43E 28ADD1 & 435929 & 1112242 & 06/23/99 & 255 & -- & -- & -- & 8.03 & -- \\
\hline 08N 44E 01CDB1 & 440237 & 1111245 & 06/30/99 & 165 & -- & -- & -- & 7.17 & -- \\
\hline \multirow[t]{2}{*}{ 08N 44E 05BBB1 } & 440322 & 1111750 & 08/09/95 & 232 & $<0.02$ & $<0.01$ & -- & 0.97 & -- \\
\hline & & & 08/09/95 & 232 & $<0.02$ & $<0.01$ & -- & 0.96 & -- \\
\hline 08N 44E 09BAA1 & 440224 & 1111605 & 06/12/98 & 40 & $<0.02$ & 0.01 & -- & 6.38 & 6.37 \\
\hline
\end{tabular}




\begin{tabular}{|c|c|c|c|c|c|c|c|c|c|}
\hline 08N 44E 10BAB1 & 440228 & 1111508 & 06/30/99 & 260 & -- & -- & -- & 0.58 & -- \\
\hline 08N 44E 17BAB1 & 440134 & 1111732 & 06/30/99 & 400 & -- & -- & -- & 5.34 & -- \\
\hline 08N 44E 26AAC1 & 435942 & 1111317 & 06/30/99 & 346 & -- & -- & -- & 3.83 & -- \\
\hline \multirow[t]{4}{*}{ 08N 44E 33BCBD1 } & 435843 & 1111634 & 08/02/96 & 342 & $<0.02$ & $<0.01$ & -- & 5.30 & -- \\
\hline & & & 08/18/97 & 342 & $<0.02$ & $<0.01$ & -- & 5.31 & -- \\
\hline & & & 08/11/98 & 342 & 0.06 & $<0.01$ & -- & 5.16 & -- \\
\hline & & & 06/16/99 & 342 & $<0.02$ & $<0.01$ & -- & 6.09 & -- \\
\hline 09N 40E 29ACB1 & 440456 & 1114555 & 06/16/99 & 479 & $<0.02$ & $<0.01$ & -- & 3.09 & -- \\
\hline \multirow[t]{2}{*}{ 09N 41E 13BBA1 } & 440653 & 1113417 & 08/10/95 & 115 & $<0.02$ & $<0.01$ & -- & 1.50 & -- \\
\hline & & & 06/23/99 & 115 & $<0.02$ & $<0.01$ & -- & 1.01 & -- \\
\hline 09N 41E 21BCC1 & 440543 & 1113805 & 08/07/97 & 415 & $<0.02$ & $<0.01$ & -- & 1.84 & -- \\
\hline \multirow[t]{2}{*}{ 09N 42E 12DCA2 } & 440704 & 1112628 & 06/09/98 & 330 & 0.05 & 0.02 & -- & 5.69 & 5.67 \\
\hline & & & $10 / 07 / 98$ & 330 & $<0.02$ & $<0.01$ & -- & 6.62 & -- \\
\hline \multirow[t]{2}{*}{ 09N 42E 21DDC1 } & 440512 & 1112953 & 07/22/97 & -- & -- & -- & -- & -- & 12.70 \\
\hline & & & 06/30/99 & -- & -- & -- & -- & 12.40 & -- \\
\hline 09N 42E 23DDA1 & 440521 & 1112725 & 06/09/98 & 85 & 0.04 & 0.01 & -- & 2.17 & 2.16 \\
\hline \multirow[t]{3}{*}{ 09N 42E 25ADC1 } & 440448 & 1112619 & 07/18/97 & 80 & -- & -- & -- & -- & 7.82 \\
\hline & & & 06/08/98 & 80 & 0.02 & 0.02 & -- & 7.23 & 7.21 \\
\hline & & & 10/06/98 & 80 & 0.02 & $<0.01$ & -- & 6.95 & -- \\
\hline \multirow[t]{3}{*}{ 09N 42E 25BAD1 } & 440458 & 1112643 & 07/18/97 & 57 & -- & -- & -- & -- & 4.77 \\
\hline & & & 06/09/98 & 57 & $<0.02$ & 0.01 & -- & 5.01 & 5.00 \\
\hline & & & $10 / 07 / 98$ & 57 & $<0.02$ & $<0.01$ & -- & 5.46 & -- \\
\hline \multirow[t]{3}{*}{ 09N 42E 25BBC1 } & 440458 & 1112715 & 08/12/97 & 161 & 0.03 & $<0.01$ & -- & 5.64 & -- \\
\hline & & & 06/08/98 & 161 & 0.05 & 0.01 & -- & 5.33 & 5.32 \\
\hline & & & $10 / 06 / 98$ & 161 & $<0.02$ & $<0.01$ & -- & 5.47 & -- \\
\hline \multirow[t]{2}{*}{ 09N 42E 25DBB1 } & 440440 & 1112640 & 06/09/98 & 80 & 0.04 & 0.01 & -- & 4.87 & 4.86 \\
\hline & & & $10 / 07 / 98$ & 80 & 0.03 & $<0.01$ & -- & 5.97 & -- \\
\hline \multirow[t]{2}{*}{ 09N 42E 26CCD1 } & 440418 & 1112820 & 07/18/97 & 57 & -- & -- & -- & -- & 4.32 \\
\hline & & & 06/09/98 & 57 & 0.02 & 0.01 & -- & 4.78 & 4.77 \\
\hline \multirow[t]{3}{*}{ 09N 42E 26CDC1 } & 440418 & 1112809 & 07/18/97 & 62 & -- & -- & -- & -- & 5.31 \\
\hline & & & 06/09/98 & 62 & $<0.02$ & 0.01 & -- & 6.57 & 6.56 \\
\hline & & & 06/30/99 & 62 & -- & -- & -- & 6.32 & -- \\
\hline \multirow[t]{2}{*}{ 09N 42E 26DAD1 } & 440432 & 1112720 & 07/18/97 & 50 & -- & -- & -- & -- & 7.44 \\
\hline & & & 06/08/98 & 50 & 0.04 & 0.01 & -- & 7.52 & 7.51 \\
\hline 09N 42E 26DDC1 & 440420 & 1112735 & 06/08/98 & 43 & 0.02 & 0.01 & -- & 3.49 & 3.48 \\
\hline \multirow[t]{2}{*}{ 09N 42E 27ABB1 } & 440505 & 1112907 & 07/22/97 & -- & -- & -- & -- & -- & 1.01 \\
\hline & & & 06/30/99 & -- & -- & -- & -- & 0.78 & -- \\
\hline
\end{tabular}




\begin{tabular}{|c|c|c|c|c|c|c|c|c|c|}
\hline 09N 42E 27ABC1 & 440458 & 1112907 & $07 / 22 / 97$ & -- & -- & -- & -- & -- & 1.74 \\
\hline \multirow[t]{2}{*}{ 09N 42E 27ACC1 } & 440445 & 1112905 & 07/22/97 & -- & -- & -- & -- & -- & 4.45 \\
\hline & & & 06/30/99 & -- & -- & -- & -- & 4.85 & -- \\
\hline \multirow[t]{2}{*}{ 09N 42E 27CDD1 } & 440418 & 1112909 & 07/22/97 & -- & -- & -- & -- & -- & 3.96 \\
\hline & & & 06/30/99 & -- & -- & -- & -- & 5.15 & -- \\
\hline 09N 42E 35AAD1 & 440411 & 1112720 & $04 / 15 / 98$ & -- & -- & -- & -- & -- & 5.27 \\
\hline \multirow[t]{3}{*}{ 09N 42E 35DDC1 } & 440328 & 1112733 & $07 / 18 / 97$ & 105 & -- & -- & -- & -- & 5.16 \\
\hline & & & $06 / 08 / 98$ & 105 & 0.02 & 0.01 & -- & 5.86 & 5.85 \\
\hline & & & 06/30/99 & 105 & -- & -- & -- & 6.50 & -- \\
\hline \multirow[t]{5}{*}{ 09N 42E 36AAB1 } & 440416 & 1112613 & $07 / 18 / 97$ & 60 & -- & -- & -- & -- & 23.50 \\
\hline & & & 06/08/98 & 60 & 0.04 & 0.02 & -- & 22.70 & 22.70 \\
\hline & & & $10 / 06 / 98$ & 60 & 0.02 & $<0.01$ & -- & 17.90 & -- \\
\hline & & & $10 / 06 / 98$ & 60 & 0.02 & $<0.01$ & 9.80 & 18.50 & -- \\
\hline & & & $06 / 25 / 99$ & 60 & 0.03 & $<0.01$ & 10.10 & 30.40 & -- \\
\hline \multirow[t]{23}{*}{ 09N 42E 36ABA1 } & 440415 & 1112625 & 02/27/95 & 289 & -- & -- & -- & -- & 7.07 \\
\hline & & & $06 / 11 / 97$ & 289 & -- & -- & -- & -- & 11.10 \\
\hline & & & $06 / 24 / 97$ & 289 & -- & -- & -- & -- & 10.80 \\
\hline & & & $06 / 24 / 97$ & 289 & -- & -- & -- & -- & 12.20 \\
\hline & & & 07/07/97 & 289 & -- & -- & -- & -- & 10.20 \\
\hline & & & $08 / 11 / 97$ & 289 & -- & -- & -- & -- & 9.05 \\
\hline & & & 09/15/97 & 289 & -- & -- & -- & -- & 8.59 \\
\hline & & & $11 / 04 / 97$ & 289 & -- & -- & -- & -- & 8.74 \\
\hline & & & $12 / 17 / 97$ & 289 & -- & -- & -- & -- & 8.95 \\
\hline & & & $01 / 12 / 98$ & 289 & -- & -- & -- & -- & 9.39 \\
\hline & & & $02 / 18 / 98$ & 289 & -- & -- & -- & -- & 9.79 \\
\hline & & & 03/19/98 & 289 & -- & -- & -- & -- & 10.20 \\
\hline & & & $04 / 14 / 98$ & 289 & -- & -- & -- & -- & 11.40 \\
\hline & & & 05/05/98 & 289 & -- & -- & -- & -- & 12.20 \\
\hline & & & 05/05/98 & 289 & -- & -- & -- & -- & 11.20 \\
\hline & & & 06/02/98 & 289 & -- & -- & -- & -- & 11.80 \\
\hline & & & 06/08/98 & 289 & 0.05 & 0.01 & -- & 11.50 & 11.50 \\
\hline & & & 07/06/98 & 289 & -- & -- & -- & -- & 11.50 \\
\hline & & & 08/05/98 & 289 & -- & -- & -- & -- & 8.66 \\
\hline & & & 09/02/98 & 289 & -- & -- & -- & -- & 7.56 \\
\hline & & & 09/03/98 & 289 & -- & -- & -- & -- & 7.56 \\
\hline & & & $10 / 06 / 98$ & 289 & $<0.02$ & $<0.01$ & 5.40 & 8.33 & -- \\
\hline & & & $10 / 06 / 98$ & 289 & -- & -- & -- & -- & 8.79 \\
\hline
\end{tabular}




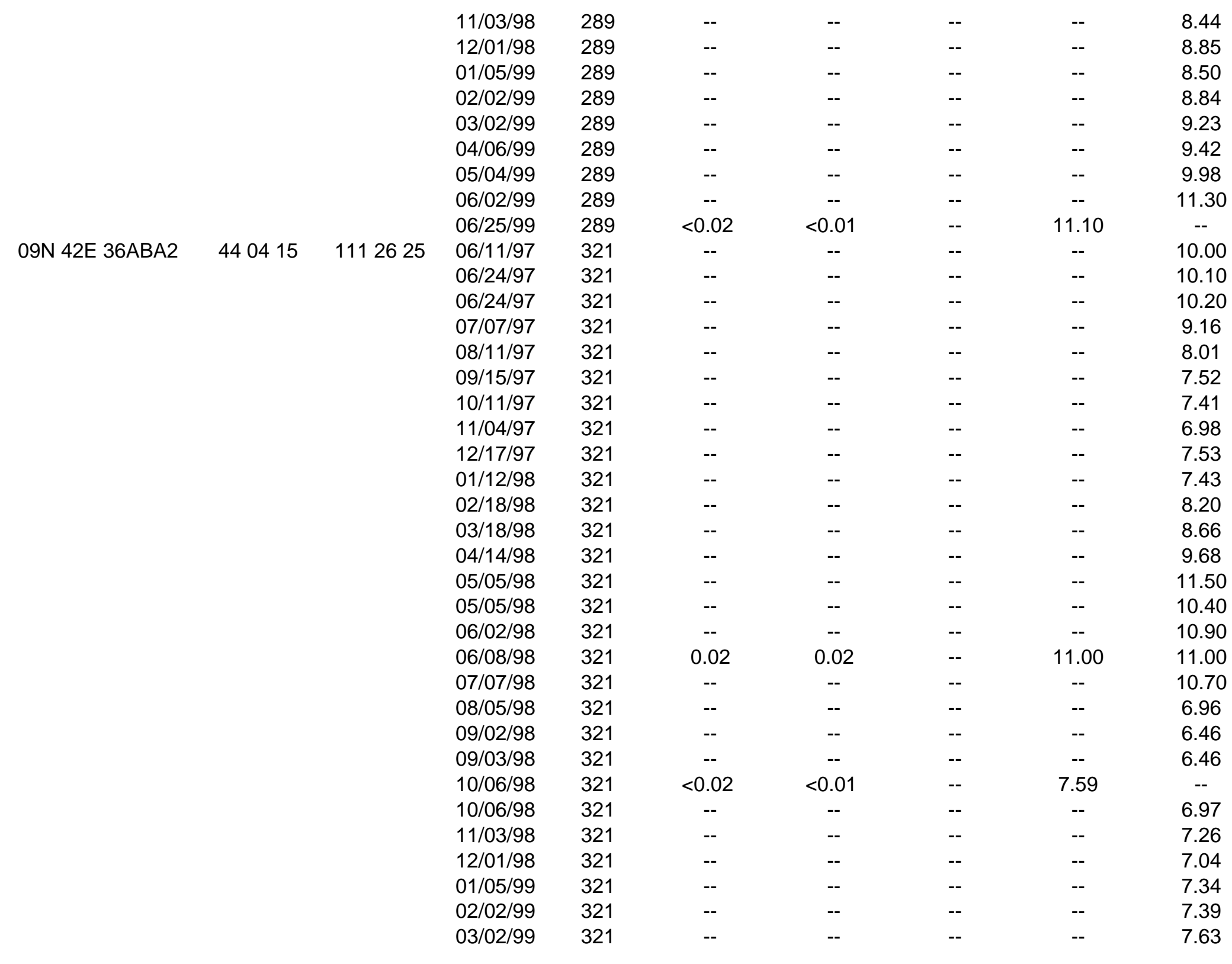




\begin{tabular}{|c|c|c|c|c|c|c|c|c|c|}
\hline & & & 04/06/99 & 321 & -- & -- & -- & -- & 8.12 \\
\hline & & & 05/04/99 & 321 & -- & -- & -- & -- & 9.58 \\
\hline & & & 06/02/99 & 321 & -- & -- & -- & -- & 9.84 \\
\hline & & & 06/25/99 & 321 & 0.03 & $<0.01$ & -- & 10.00 & -- \\
\hline \multirow[t]{3}{*}{ 09N 42E 36CAA1 } & 440350 & 1112646 & 07/18/97 & 50 & -- & -- & -- & -- & 5.89 \\
\hline & & & 06/08/98 & 50 & 0.02 & 0.02 & -- & 6.35 & 6.34 \\
\hline & & & 10/07/98 & 50 & $<0.02$ & $<0.01$ & -- & 5.91 & -- \\
\hline \multirow[t]{3}{*}{ 09N 42E 36CBB1 } & 440350 & 1112717 & 07/18/97 & 55 & -- & -- & -- & -- & 5.38 \\
\hline & & & 06/08/98 & 55 & 0.05 & 0.01 & -- & 6.05 & 6.04 \\
\hline & & & 06/08/98 & 55 & -- & -- & -- & 6.04 & -- \\
\hline \multirow[t]{2}{*}{ 09N 42E 36DAA1 } & 440347 & 1112607 & 06/08/98 & 90 & 0.05 & 0.02 & -- & 5.42 & 5.40 \\
\hline & & & $10 / 06 / 98$ & 90 & $<0.02$ & $<0.01$ & -- & 5.01 & -- \\
\hline 09N 43E 13ABB1 & 440648 & 1111918 & 06/11/98 & 280 & 0.03 & 0.01 & -- & 0.35 & 0.34 \\
\hline 09N 43E 14ADA1 & 440636 & 1112015 & 06/09/98 & 40 & 0.06 & 0.01 & -- & 1.09 & 1.08 \\
\hline 09N 43E 14DDC1 & 440605 & 1112021 & 06/09/98 & 321 & 0.04 & 0.01 & -- & 5.59 & 5.57 \\
\hline \multirow[t]{2}{*}{ 09N 43E 16DAB1 } & 440620 & 1112240 & 06/09/98 & 380 & 0.04 & 0.01 & -- & 8.40 & 8.38 \\
\hline & & & $10 / 07 / 98$ & 380 & $<0.02$ & $<0.01$ & -- & 8.97 & -- \\
\hline 09N 43E 19BCB1 & 440545 & 1112603 & 06/10/98 & 2264 & $<0.02$ & 0.01 & -- & 0.28 & 0.26 \\
\hline \multirow[t]{4}{*}{ 09N 43E 19BCC1 } & 440536 & 1112605 & 07/18/97 & 80 & -- & -- & -- & -- & 11.90 \\
\hline & & & 06/10/98 & 80 & $<0.02$ & 0.01 & -- & 9.89 & 9.88 \\
\hline & & & 06/11/98 & 80 & 0.02 & 0.01 & -- & 9.48 & 9.47 \\
\hline & & & 10/07/98 & 80 & $<0.02$ & $<0.01$ & -- & 13.30 & -- \\
\hline \multirow[t]{2}{*}{ 09N 43E 19CCC1 } & 440515 & 1112605 & 07/18/97 & -- & -- & -- & -- & -- & 4.65 \\
\hline & & & 06/09/98 & -- & 0.05 & 0.02 & -- & 4.96 & 4.94 \\
\hline \multirow[t]{3}{*}{ 09N 43E 19CDA1 } & 440519 & 1112531 & 07/18/97 & 37 & -- & -- & -- & -- & 11.50 \\
\hline & & & 06/08/98 & 37 & 0.05 & 0.01 & -- & 11.60 & 11.60 \\
\hline & & & 10/07/98 & 37 & $<0.02$ & $<0.01$ & -- & 8.49 & -- \\
\hline \multirow[t]{3}{*}{ 09N 43E 19CDB1 } & 440521 & 1112548 & 07/18/97 & 127 & -- & -- & -- & -- & 4.78 \\
\hline & & & 06/08/98 & 127 & 0.05 & 0.01 & -- & 7.08 & 7.06 \\
\hline & & & 06/08/98 & 127 & 0.04 & 0.01 & -- & 7.17 & 7.15 \\
\hline \multirow[t]{3}{*}{ 09N 43E 19DAD1 } & 440523 & 1112455 & 07/18/97 & 180 & -- & -- & -- & -- & 9.51 \\
\hline & & & 06/09/98 & 180 & 0.06 & 0.01 & -- & 11.00 & 10.90 \\
\hline & & & 10/07/98 & 180 & $<0.02$ & $<0.01$ & -- & 10.30 & -- \\
\hline 09N 43E 19DBC1 & 440523 & 1112523 & 06/09/98 & 222 & $<0.02$ & 0.01 & -- & 9.09 & 9.08 \\
\hline \multirow[t]{3}{*}{ 09N 43E 21CCC1 } & 440511 & 1112334 & 06/11/98 & 156 & $<0.02$ & 0.01 & -- & 12.20 & 12.20 \\
\hline & & & 10/05/98 & 156 & 0.06 & 0.01 & -- & 13.20 & 13.20 \\
\hline & & & $10 / 05 / 98$ & 156 & $<0.02$ & $<0.01$ & -- & 12.80 & -- \\
\hline
\end{tabular}




\begin{tabular}{|c|c|c|c|c|c|c|c|c|c|}
\hline \multirow{2}{*}{ 09N 43E 21CCC2 } & 440512 & 1112339 & 07/18/97 & -- & -- & -- & -- & -- & 29.80 \\
\hline & & & 06/08/98 & -- & 0.04 & 0.02 & -- & 19.60 & 19.60 \\
\hline \multirow[t]{3}{*}{ 09N 43E 21CDD1 } & 440510 & 1112310 & $07 / 18 / 97$ & 237 & -- & -- & -- & -- & 9.56 \\
\hline & & & 06/09/98 & 237 & 0.04 & 0.01 & -- & 10.30 & 10.30 \\
\hline & & & $10 / 05 / 98$ & 237 & $<0.02$ & $<0.01$ & -- & 9.72 & -- \\
\hline 09N 43E 22BCC1 & 440541 & 1112227 & 06/30/99 & 302 & -- & -- & -- & 7.74 & -- \\
\hline \multirow[t]{3}{*}{ 09N 43E 23AAB1 } & 440557 & 1112020 & 08/02/96 & 342 & $<0.02$ & $<0.01$ & -- & 7.90 & -- \\
\hline & & & 06/10/98 & 342 & 0.03 & 0.01 & -- & 7.15 & 7.13 \\
\hline & & & 10/06/98 & 342 & $<0.02$ & $<0.01$ & 4.30 & 7.28 & -- \\
\hline 09N 43E 25AAA1 & 440507 & 1111848 & 06/11/98 & 170 & $<0.02$ & $<0.01$ & -- & 5.11 & -- \\
\hline \multirow[t]{3}{*}{ 09N 43E 25AAA2 } & 440503 & 1111844 & 07/18/97 & 218 & -- & -- & -- & -- & 8.36 \\
\hline & & & 06/09/98 & 218 & $<0.02$ & 0.01 & -- & 7.58 & 7.57 \\
\hline & & & 06/09/98 & 218 & 0.03 & 0.01 & -- & 7.69 & 7.68 \\
\hline 09N 43E 25DCC1 & 440417 & 1111924 & 06/11/98 & -- & $<0.02$ & 0.01 & -- & 7.77 & 7.76 \\
\hline 09N 43E 26BBC1 & 440457 & 1112115 & 06/09/98 & 400 & 0.02 & 0.01 & -- & 10.40 & 10.40 \\
\hline 09N 43E 27BAB1 & 440507 & 1112204 & 06/09/98 & -- & $<0.02$ & -- & -- & 10.70 & -- \\
\hline 09N 43E 28ADB1 & 440452 & 1112245 & 06/09/98 & 202 & 0.02 & 0.01 & -- & 12.90 & 12.90 \\
\hline \multirow[t]{2}{*}{ 09N 43E 28DAB1 } & 440437 & 1112245 & 07/18/97 & 157 & -- & -- & -- & -- & 6.78 \\
\hline & & & 06/09/98 & 157 & 0.05 & 0.01 & -- & 6.62 & 6.61 \\
\hline \multirow[t]{4}{*}{ 09N 43E 28DAC1 } & 440431 & 1112245 & 08/02/96 & 198 & $<0.02$ & $<0.01$ & -- & 6.20 & -- \\
\hline & & & 07/22/97 & 198 & -- & -- & -- & -- & 5.92 \\
\hline & & & 06/09/98 & 198 & 0.03 & 0.01 & -- & 5.76 & 5.75 \\
\hline & & & $10 / 05 / 98$ & 198 & $<0.02$ & $<0.01$ & -- & 5.47 & -- \\
\hline \multirow[t]{2}{*}{ 09N 43E 29BBB1 } & 440508 & 1112452 & 06/10/98 & 100 & 0.02 & 0.01 & -- & 5.01 & 5.00 \\
\hline & & & 10/05/98 & 100 & $<0.02$ & $<0.01$ & -- & 4.61 & -- \\
\hline \multirow[t]{3}{*}{ 09N 43E 29DCC1 } & 440419 & 1112412 & 07/18/97 & 122 & -- & -- & -- & -- & 8.56 \\
\hline & & & 06/09/98 & 122 & $<0.02$ & 0.01 & -- & 8.24 & 8.23 \\
\hline & & & 10/05/98 & 122 & $<0.02$ & $<0.01$ & -- & 11.50 & -- \\
\hline \multirow[t]{2}{*}{ 09N 43E 29DDD1 } & 440418 & 1112345 & 07/18/97 & -- & -- & -- & -- & -- & 7.86 \\
\hline & & & 06/09/98 & -- & 0.03 & 0.01 & -- & 7.55 & 7.54 \\
\hline \multirow{2}{*}{ 09N 43E 30BCC1 } & 440444 & 1112604 & 06/11/98 & 105 & 0.03 & $<0.01$ & -- & 5.40 & -- \\
\hline & & & $10 / 06 / 98$ & 105 & $<0.02$ & $<0.01$ & -- & 5.54 & -- \\
\hline \multirow[t]{5}{*}{ 09N 43E 30CCB2 } & 440426 & 1112602 & $02 / 21 / 96$ & 120 & -- & -- & -- & -- & 5.87 \\
\hline & & & 02/06/97 & 120 & -- & -- & -- & -- & 6.99 \\
\hline & & & 03/09/98 & 120 & -- & -- & -- & -- & 5.37 \\
\hline & & & 06/11/98 & 120 & 0.03 & $<0.01$ & -- & 8.90 & -- \\
\hline & & & $10 / 06 / 98$ & 120 & 0.02 & $<0.01$ & -- & 8.38 & -- \\
\hline
\end{tabular}




\begin{tabular}{|c|c|c|c|c|c|c|c|c|c|}
\hline \multirow[t]{3}{*}{ 09N 43E 30CCC2 } & 440418 & 1112559 & $06 / 08 / 98$ & 73 & 0.05 & 0.01 & -- & 14.30 & 14.20 \\
\hline & & & 06/08/98 & 73 & 0.05 & 0.01 & -- & 14.20 & 14.10 \\
\hline & & & $10 / 06 / 98$ & 73 & 0.02 & $<0.01$ & -- & 9.44 & -- \\
\hline \multirow[t]{2}{*}{ 09N 43E 30CDC1 } & 440418 & 1112544 & $07 / 18 / 97$ & 74 & -- & -- & -- & -- & 3.79 \\
\hline & & & $06 / 10 / 98$ & 74 & 0.03 & 0.01 & -- & 3.76 & 3.75 \\
\hline \multirow[t]{2}{*}{ 09N 43E 30CDD1 } & 440420 & 1112531 & 07/18/97 & -- & -- & -- & -- & -- & 3.87 \\
\hline & & & $06 / 09 / 98$ & -- & $<0.02$ & 0.01 & -- & 4.45 & 4.44 \\
\hline \multirow[t]{2}{*}{ 09N 43E 30DAA1 } & 440437 & 1112459 & $06 / 09 / 98$ & 140 & $<0.02$ & 0.01 & -- & 4.87 & 4.86 \\
\hline & & & $10 / 05 / 98$ & 140 & 0.02 & $<0.01$ & -- & 2.34 & -- \\
\hline 09N 43E 30DAB1 & 440438 & 1112504 & $07 / 18 / 97$ & -- & -- & -- & -- & -- & 4.71 \\
\hline 09N 43E 30DAC1 & 440431 & 1112510 & $07 / 18 / 97$ & -- & -- & -- & -- & -- & 2.60 \\
\hline \multirow[t]{2}{*}{ 09N 43E 30DBD2 } & 440435 & 1112520 & 07/18/97 & 48 & -- & -- & -- & -- & 2.86 \\
\hline & & & $06 / 11 / 98$ & 48 & 0.03 & 0.01 & -- & 4.80 & 4.79 \\
\hline \multirow[t]{3}{*}{ 09N 43E 30DCA1 } & 440425 & 1112512 & 07/18/97 & 38 & -- & -- & -- & -- & 6.74 \\
\hline & & & $06 / 09 / 98$ & 38 & 0.03 & 0.01 & -- & 6.53 & 6.52 \\
\hline & & & $10 / 06 / 98$ & 38 & $<0.02$ & $<0.01$ & -- & 6.27 & -- \\
\hline 09N 43E 30DCB3 & 440425 & 1112528 & $06 / 11 / 98$ & 50 & $<0.02$ & 0.01 & -- & 5.69 & 5.68 \\
\hline 09N 43E 30DCD3 & 440419 & 1112513 & $06 / 10 / 98$ & 45 & 0.02 & 0.01 & -- & 5.72 & 5.71 \\
\hline \multirow[t]{3}{*}{ 09N 43E 30DDA1 } & 440426 & 1112501 & 07/18/97 & 45 & -- & -- & -- & -- & 9.79 \\
\hline & & & $06 / 10 / 98$ & 45 & 0.03 & 0.01 & -- & 6.61 & 6.60 \\
\hline & & & $06 / 10 / 98$ & 45 & 0.03 & 0.01 & -- & 6.53 & 6.51 \\
\hline \multirow[t]{2}{*}{ 09N 43E 30DDB1 } & 440424 & 1112504 & $07 / 18 / 97$ & -- & -- & -- & -- & -- & 5.65 \\
\hline & & & $06 / 10 / 98$ & -- & 0.02 & 0.01 & -- & 5.22 & 5.21 \\
\hline \multirow{2}{*}{ 09N 43E 30DDB2 } & & & $07 / 18 / 97$ & 55 & -- & -- & -- & -- & 6.07 \\
\hline & & & $06 / 10 / 98$ & 55 & $<0.02$ & 0.01 & -- & 5.67 & 5.66 \\
\hline \multirow[t]{3}{*}{ 09N 43E 30DDB3 } & 440429 & 1112505 & $07 / 18 / 97$ & 35 & -- & -- & -- & -- & 8.97 \\
\hline & & & $06 / 10 / 98$ & 35 & 0.02 & 0.01 & -- & 6.57 & 6.55 \\
\hline & & & $10 / 05 / 98$ & 35 & $<0.02$ & $<0.01$ & -- & 7.06 & -- \\
\hline \multirow[t]{3}{*}{ 09N 43E 30DDB4 } & & & 07/18/97 & 65 & -- & -- & -- & -- & 0.98 \\
\hline & & & $06 / 10 / 98$ & 65 & 0.02 & 0.01 & -- & 4.77 & 4.75 \\
\hline & & & $10 / 05 / 98$ & 65 & 0.02 & $<0.01$ & -- & 3.69 & -- \\
\hline 09N 43E 30DDB5 & 440426 & 1112504 & $06 / 10 / 98$ & 62 & $<0.02$ & 0.01 & -- & 5.83 & 5.82 \\
\hline 09N 43E 30DDB6 & & & 06/10/98 & 50 & 0.03 & 0.01 & -- & 6.60 & 6.59 \\
\hline \multirow[t]{3}{*}{ 09N 43E 30DDC1 } & 440418 & 1112503 & 07/18/97 & 40 & -- & -- & -- & -- & 7.47 \\
\hline & & & 06/09/98 & 40 & 0.02 & 0.01 & -- & 8.71 & 8.70 \\
\hline & & & $10 / 07 / 98$ & 40 & $<0.02$ & $<0.01$ & -- & 8.51 & -- \\
\hline 09N 43E 31BAB1 & 440415 & 1112542 & 07/18/97 & 100 & -- & -- & -- & -- & 7.49 \\
\hline
\end{tabular}




\begin{tabular}{|c|c|c|c|c|c|c|c|c|c|}
\hline & & & 06/08/98 & 100 & 0.06 & 0.01 & -- & 8.92 & 8.91 \\
\hline \multirow[t]{3}{*}{ 09N 43E 31DCD1 } & 440329 & 1112513 & 07/18/97 & 58 & -- & -- & -- & -- & 5.66 \\
\hline & & & 06/10/98 & 58 & $<0.02$ & 0.01 & -- & 5.41 & 5.40 \\
\hline & & & $10 / 07 / 98$ & 58 & $<0.02$ & $<0.01$ & -- & 5.78 & -- \\
\hline \multirow[t]{2}{*}{ 09N 43E 32ABB1 } & 440415 & 1112416 & $07 / 18 / 97$ & -- & -- & -- & -- & -- & 6.43 \\
\hline & & & 06/08/98 & -- & 0.02 & 0.02 & -- & 7.37 & 7.35 \\
\hline \multirow[t]{2}{*}{ 09N 43E 32BAB1 } & 440415 & 1112434 & $07 / 22 / 97$ & 130 & -- & -- & -- & -- & 8.82 \\
\hline & & & $06 / 11 / 98$ & 130 & $<0.02$ & 0.01 & -- & 8.78 & 8.77 \\
\hline \multirow[t]{3}{*}{ 09N 43E 33AAA1 } & 440415 & 1112236 & 07/18/97 & -- & -- & -- & -- & -- & 5.05 \\
\hline & & & $06 / 10 / 98$ & -- & 0.02 & 0.01 & -- & 4.80 & 4.79 \\
\hline & & & $06 / 10 / 98$ & -- & 0.02 & 0.01 & -- & 5.17 & 5.16 \\
\hline 09N 43E 33ABA1 & 440445 & 1112247 & 06/30/99 & 205 & -- & -- & -- & 5.12 & -- \\
\hline \multirow[t]{4}{*}{ 09N 43E 33CDD1 } & 440326 & 1112312 & 07/18/97 & 180 & -- & -- & -- & -- & 9.21 \\
\hline & & & 06/09/98 & 180 & 0.03 & 0.01 & -- & 7.96 & 7.95 \\
\hline & & & $10 / 06 / 98$ & 180 & $<0.02$ & $<0.01$ & -- & 9.01 & -- \\
\hline & & & 06/30/99 & 180 & -- & -- & -- & 6.76 & -- \\
\hline 09N 43E 33DCD1 & 440325 & 1112255 & $06 / 11 / 98$ & -- & 0.02 & 0.01 & -- & 3.86 & 3.85 \\
\hline \multirow[t]{2}{*}{ 09N 43E 34CDC1 } & 440326 & 1112206 & $07 / 18 / 97$ & 275 & -- & -- & -- & -- & 0.79 \\
\hline & & & $06 / 09 / 98$ & 275 & $<0.02$ & 0.01 & -- & 0.87 & 0.86 \\
\hline 09N 43E 34CDD1 & 440326 & 1112159 & $06 / 30 / 99$ & 185 & -- & -- & -- & 2.62 & -- \\
\hline 09N 43E 34DAA1 & 440348 & 1112117 & $06 / 10 / 98$ & 104 & 0.04 & 0.01 & -- & 16.40 & 16.40 \\
\hline \multirow[t]{2}{*}{ 09N 43E 35AAB1 } & 440415 & 1112020 & $06 / 10 / 98$ & 32 & 0.02 & 0.01 & -- & 12.30 & 12.30 \\
\hline & & & $10 / 06 / 98$ & 32 & $<0.02$ & $<0.01$ & -- & 6.87 & -- \\
\hline \multirow[t]{3}{*}{ 09N 43E 35CBB1 } & 440349 & 1112115 & 07/18/97 & 114 & -- & -- & -- & -- & 9.39 \\
\hline & & & $06 / 10 / 98$ & 114 & $<0.02$ & 0.01 & -- & 15.40 & 15.40 \\
\hline & & & 06/18/99 & 114 & -- & -- & -- & 24.90 & -- \\
\hline \multirow[t]{4}{*}{ 09N 44E 08BDA1 } & 440727 & 1111703 & $06 / 11 / 98$ & 420 & 0.14 & 0.01 & -- & 0.26 & 0.25 \\
\hline & & & $06 / 11 / 98$ & 420 & 0.14 & 0.01 & -- & 0.26 & 0.25 \\
\hline & & & $10 / 07 / 98$ & 420 & $<0.02$ & $<0.01$ & -- & 0.23 & -- \\
\hline & & & $10 / 07 / 98$ & 420 & $<0.02$ & $<0.01$ & -- & 0.23 & -- \\
\hline 09N 44E 15CAC1 & 440616 & 1111448 & $06 / 11 / 98$ & 360 & 0.03 & 0.01 & -- & 0.16 & 0.15 \\
\hline 09N 44E 17ABC1 & 440645 & 1111656 & $06 / 11 / 98$ & 421 & 0.05 & 0.01 & -- & 0.29 & 0.28 \\
\hline 09N 44E 21CCC1 & 440510 & 1111620 & 06/10/98 & 100 & 0.04 & 0.01 & -- & 7.71 & 7.70 \\
\hline \multirow[t]{4}{*}{ 09N 44E 23CAB1 } & 440529 & 1111335 & $07 / 12 / 95$ & 120 & -- & -- & -- & -- & 0.36 \\
\hline & & & $07 / 01 / 96$ & 120 & -- & -- & -- & -- & 0.24 \\
\hline & & & $06 / 10 / 98$ & 120 & 0.03 & 0.01 & -- & 0.28 & 0.26 \\
\hline & & & $10 / 05 / 98$ & 120 & $<0.02$ & $<0.01$ & -- & 0.28 & -- \\
\hline
\end{tabular}




\begin{tabular}{|c|c|c|c|c|c|c|c|c|c|}
\hline & & & 06/18/99 & 120 & -- & -- & -- & 0.29 & -- \\
\hline 09N 44E 29AAA1 & 440507 & 1111626 & $06 / 10 / 98$ & 84 & 0.03 & 0.01 & -- & 7.22 & 7.21 \\
\hline \multirow[t]{4}{*}{ 09N 44E 30CBB1 } & 440438 & 1111846 & 08/12/97 & 180 & 0.03 & $<0.01$ & -- & 18.00 & -- \\
\hline & & & 06/10/98 & 180 & 0.02 & 0.01 & -- & 20.50 & 20.50 \\
\hline & & & $10 / 06 / 98$ & 180 & 0.03 & $<0.01$ & -- & 14.30 & -- \\
\hline & & & 06/18/99 & 180 & -- & -- & -- & 20.30 & -- \\
\hline 09N 45E 12BAC1S & 440733 & 1110517 & 06/10/98 & -- & $<0.02$ & 0.01 & -- & 0.12 & 0.11 \\
\hline \multirow[t]{2}{*}{ 09N 45E 17CBB1 } & 440624 & 1111024 & 07/11/95 & 185 & -- & -- & -- & -- & 0.14 \\
\hline & & & $06 / 10 / 98$ & 185 & $<0.02$ & 0.01 & -- & 0.18 & 0.17 \\
\hline 10N 41E 34ADA1 & 440915 & 1113549 & $08 / 14 / 97$ & 160 & $<0.02$ & 0.01 & -- & $<0.05$ & -- \\
\hline 10N 41E 35CBD1 & 440853 & 1113536 & 08/05/96 & 175 & 0.04 & $<0.01$ & -- & $<0.05$ & -- \\
\hline \multirow[t]{2}{*}{ 10N 44E 10CBA1S } & 441219 & 1111457 & 08/14/97 & -- & $<0.02$ & $<0.01$ & -- & 0.14 & -- \\
\hline & & & $06 / 11 / 98$ & -- & $<0.02$ & 0.01 & -- & 0.12 & 0.11 \\
\hline 10N 45E 26DCA2 & 440938 & 1110600 & $06 / 10 / 98$ & 405 & 0.05 & 0.01 & -- & 0.07 & 0.06 \\
\hline \multirow[t]{5}{*}{$11 \mathrm{~N} 42 \mathrm{E} 12 \mathrm{CBCA} 1$} & 441737 & 1112714 & $08 / 11 / 95$ & 123 & $<0.02$ & $<0.01$ & -- & 1.10 & -- \\
\hline & & & $08 / 08 / 96$ & 123 & 0.02 & $<0.01$ & -- & 0.97 & -- \\
\hline & & & 08/15/97 & 123 & $<0.02$ & $<0.01$ & -- & 1.24 & -- \\
\hline & & & 08/11/98 & 123 & 0.06 & $<0.01$ & -- & 1.09 & -- \\
\hline & & & 08/24/99 & 123 & $<0.02$ & $<0.01$ & -- & 1.29 & -- \\
\hline $11 \mathrm{~N} 43 \mathrm{E} 28 \mathrm{CAA} 2$ & 441442 & 1112318 & 08/11/95 & 298 & $<0.02$ & $<0.01$ & -- & 0.53 & -- \\
\hline 12N 43E 17DBA1 & 442151 & 1112403 & 08/08/96 & 65 & 0.02 & $<0.01$ & -- & 0.37 & -- \\
\hline \multirow[t]{3}{*}{$12 \mathrm{~N} 44 \mathrm{E} 17 \mathrm{ACA} 1$} & 442205 & 1111642 & 09/14/95 & 140 & $<0.02$ & $<0.01$ & -- & 0.08 & -- \\
\hline & & & 08/10/99 & 140 & $<0.02$ & $<0.01$ & -- & 0.09 & -- \\
\hline & & & 08/10/99 & 140 & $<0.02$ & $<0.01$ & -- & 0.09 & -- \\
\hline 48N 118W 10CCC1 & 440750 & 1110050 & 06/11/98 & 20 & 0.02 & 0.01 & -- & 0.10 & 0.09 \\
\hline
\end{tabular}

